\title{
草酸酯加氢制乙二醇钉金属均相催化体系的研究进展
}

\author{
张亦伟 \\ 陈艺林方霄龙 \\ 袁友珠* \\ 朱红平* \\ (厦门大学化学化工学院 固体表面物理化学国家重点实验室 醇醚酯清洁生产国家工程实验室 厦门 361005)
}

\begin{abstract}
摘要 草酸酯加氢是重要的有机化学反应，在工业制乙二醇生产中有着重要的应用. 对钉金属配合物均相催化反应的 研究进行了综述. 以催化反应体系为焦点，探讨了多种因素如温度、氢气压力、催化剂浓度、反应时间、添加剂等对 底物转化率以及产物选择性的影响, 并讨论了催化反应机理, 其中金属-配体协同促进的 $\mathrm{H}_{2}$ 分子异裂, 进而完成对底 物分子中酯基依次加氢的反应机理是探讨的重点, 这为新型催化剂的设计和应用提供参考.

关键词 草酸酯加氢; 钉金属均相催化体系; 乙二醇; 反应条件; 催化机理
\end{abstract}

\section{Advances for the Ruthenium Complexes-Based Homogeneous Catalytic Hydrogenation of Oxalates to Ethylene Glycol}

\author{
Zhang, Yiwei Chen, Yilin Fang, Xiaolong Yuan, Youzhu* Zhu, Hongping* \\ (State Key Laboratory of Physical Chemistry of Solid Surfaces, National Engineering Laboratory for Green Chemical Produc- \\ tions of Alcohols-Ethers-Esters, College of Chemistry and Chemical Engineering, Xiamen University, Xiamen 361005)
}

\begin{abstract}
Hydrogenation of oxalates is one of the important organic reactions, which has an ultimate use for the industrial production of ethylene glycol. The studies on the ruthenium complexes-based homogeneous catalytic reaction systems are herein summarized. With the focus on the catalytic reaction systems, the important factors with significant influences on the oxalate transformation efficiency as well as the product selectivity are discussed, including temperature, $\mathrm{H}_{2}$ pressure, catalyst concentration, reaction duration, additives, and so on. The catalytic reaction mechanisms are also discussed in detail, where the mechanism for the $\mathrm{H}_{2}$-heterolysis promoted under the metal-ligand cooperation for the oxalate hydrogenation to ethylene glycol is enhanced. This study would be useful for designing the new catalyst applicable in industry.

Keywords hydrogenation of oxalate; ruthenium complexes-based homogeneous catalytic system; ethylene glycol; reaction conditions; catalytic mechanism
\end{abstract}

乙二醇(EG)是一种重要的基础有机化工原料, 用于 防冻剂和溶剂. 它也是精细化学品和药物合成用的原料 以及聚酯和聚醚合成用的重要单体等 ${ }^{[1]}$. 近年来 $95 \%$ 的 乙二醇用于生产聚对苯二甲酸乙二醇酯(PET). 2015 年 的统计数据表明全球乙二醇的产能为 3100 万吨, 产量 达 2700 万吨，而我国乙二醇的表观消费量接近 1430 万 吨. 至 2030 年, 全球对乙二醇产品的需求仍会保持一定 的增长态势 ${ }^{[2]}$. 近年来合成乙二醇的研究受到了很多科 研工作者的关注, 聚焦点是工业化生产技术以及新型反 应路线等.

乙二醇的合成路线主要包括以乙烯为原料的环氧 乙烷法和以合成气为原料的草酸酯法. 环氧乙烷法涉及
乙烯在银催化剂的作用下与氧气发生环加成反应生成 环氧乙烷，以及环氧乙烷进一步在酸或碱催化剂的作用 下水合转化成乙二醇(Scheme 1). 目前该法的生产技术 主要由英荷壳牌(Shell)公司、美国科学设计(SD)公司和 陶氏化学(DOW)公司掌握 ${ }^{[2]}$. 草酸酯法又称合成气氧化 偶联法, 如 Scheme 2 所示, 第一步由甲醇、一氧化氮和 氧气反应生成亚硝酸甲酯; 第二步亚硝酸甲酯与一氧化 碳在钯催化剂作用下反应生成草酸二甲酯(DMO), 并释 放出一氧化氮; 第三步草酸二甲酯在铜-铬或铜催化剂 (注: 工业用催化剂)作用下加氢转化成乙二醇, 并释放 出甲醇. 该路线由美国 UCC 公司于 1966 年提出, 1978 年日本宇部兴产对该技术进行了改进，近年来国内多家

\footnotetext{
* Corresponding authors. E-mail: yzyuan@xmu.edu.cn; hpzhu@xmu.edu.cn Received March 10, 2017; revised May 2, 2017; published online May 17, 2017.
}

Project supported by the National Natural Science Foundation of China (Nos. 21473142, 91545115, 21473145) and the Innovative Research Team of China (Nos. IRT 14R31, J1310024).

国家自然科学基金(Nos. 21473142, 91545115, 21473145)和教育部创新团队(Nos. IRT_14R31, J1310024)资助项目. 
单位对该技术进行了革新，并积极推向工业化生产 ${ }^{[2]}$.

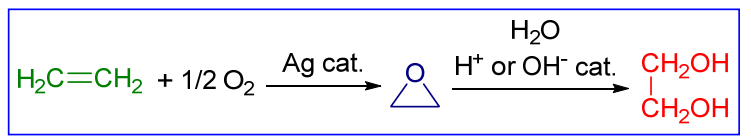

图式 1 环氧乙烷法合成乙二醇的反应路线

Scheme 1 Ethylene epoxide-mediated reaction route to ethylene glycol

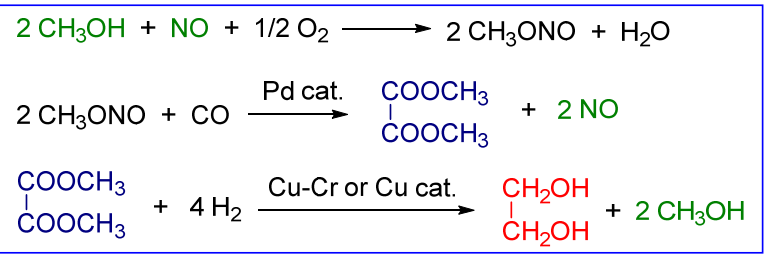

图式 2 草酸酯法合成乙二醇的反应路线

Scheme 2 DMO-mediated reaction route to ethylene glycol

环氧乙烷法生产乙二醇是传统的石油化工路线, 其 中原料乙烯依赖于石油的裂解. 草酸酯法生产乙二醇则 是现代煤化工路线. 从 Scheme 2 的反应路线看, 一氧化 氮和甲醇可以循环利用, 一氧化氮通过氨气和氧气反应 制备, 氨气来源于合成气中的氢气与氮气经铁催化剂合 成; 甲醇则可以经合成气工业生产. 因此, 整个反应完 全可以以合成气为原料. 近年来, 合成气制烯烃产业得 到很大发展, 其中产出的乙烯也可用于环氧乙烷法的原 料, 也就是说环氧乙烷法也可以经煤化工路线实现. 总 的来说, 从化学原理角度看, 这两种路线都是热力学和 动力学可行的反应, 并且原料来源丰富; 但从商业经济 角度考虑, 这两种路线孰优孰劣则需要考察煤和石油资 源的市场价格以及生产和技术成本. 环氧乙烷法反应过 程相对简单, 而草酸酯法则要复杂得多. 后者的研究是 近年来的热点.

在草酸酯法中, 一氧化碳与亚硝酸酯钯催化偶联是 合成草酸酯的关键步骤, 但是草酸酯加氢是合成乙二醇 的必要过程. 草酸酯是一种特殊的双酯基碳碳键联的分 子, 其加氢转化为醇是一种重要的酯类化合物的氢解反

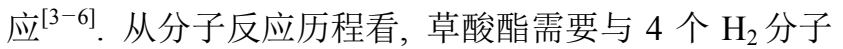
反应，最后生成一个分子的乙二醇和两个分子的单醇, 这是一个非常复杂的反应过程. 如 Scheme 3 所示, 草酸 二甲酯中的一个酯基与 $\mathrm{H}_{2}$ 在催化剂的作用下发生羰基 键 $(\mathrm{C}=\mathrm{O})$ 加成生成半缩醛, 半缩醛不稳定重排脱去一 个甲醇分子生成乙醛酸甲酯; 乙醛酸甲酯中的醛基进一 步与 $\mathrm{H}_{2}$ 在催化剂的作用下发生 $\mathrm{C}=\mathrm{O}$ 键加成生成乙醇 酸甲酯(MG). 乙醇酸甲酯中的酯基经过类似的加氢过 程生成乙二醇( $\mathrm{EG})$. 在整个反应过程中两种半缩醛分子 以及乙醛酸甲酯和 $\beta$-羟基乙醛均不稳定，可看作是中间 体; 但是乙醇酸甲酯是稳定的分子, 可以从反应中分离.
因为草酸二甲酯和乙醇酸甲酯的加氢反应性有一定的 差异性，同时反应过程中常有副反应发生，因此，除了 催化反应活性外，反应选择性实际上也是评定催化反应 体系好坏的一个重要因素, 这一点往往不被重视。

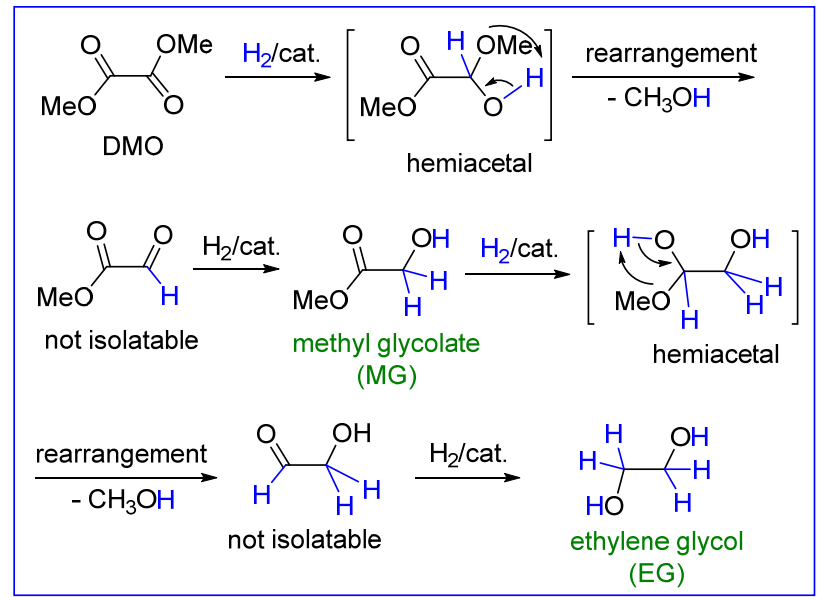

图式 3 草酸二甲酯加氢生成乙二醇的基元反应过程 Scheme 3 Elemental reactions for hydrogenation of DMO to EG

用于草酸酯加氢反应的催化剂主要包括无机物负 载型金属催化剂和金属配合物催化剂两大类，前者用于 非均相反应，后者则在均相反应体系中使用，无论哪一 种催化剂, 均需要经过如 Scheme 3 所示的反应历程. 已 经有一些文章对前者进行了综述讨论 ${ }^{[7]}$ ，但是对后者的 综述甚少. 目前工业用催化剂集中于负载型，但是由于 负载型催化剂的复杂结构以及非均相反应特征，研究提 出的催化反应机理存在多种争论. 相比较而言, 均相催 化体系中的催化剂具有明确的结构组成，而且整个体系 反应条件温和，易于实施，可以通过多种测试手段对反 应过程进行跟踪分析. 这些使得相关反应机理的研究具 有可行性和合理性. 尽管如此, 针对 Scheme 3 所示的复 杂转化, 化学家们至今对其中的反应机理尚无明确的定 论. 本文将从 20 世纪 80 年代草酸酯加氢钓金属均相催 化反应体系的最初发现到目前的研究状况逐一论述，重 点介绍典型的催化剂体系, 并探讨反应条件对产物的影 响以及催化反应机理.

\section{1 草酸酯加氢制备乙二醇的钉金属均相催化体} 系

早期的研究显示酯类分子加氢生成醇主要使用氢 化试剂, 如氢化锂铝、嗍氢化钠等, 反应后需要进一步 水解形成产物，但是该过程要消耗计量的氢化试剂，同 时产生金属氢氧化物或氧化物或盐等废弃物 ${ }^{[3 \sim 6,8 \sim 9]}$. 因 此这种方法在初期探索方面有一定的积极意义，但不具 有应用价值. 负载型的金属催化剂，如二氧化硅负载的 
铜一铬、铜等相继被发现, 但是加氢反应条件苛刻, 如早 期报道的反应温度达到 $200 \sim 250{ }^{\circ} \mathrm{C}$, 压力达到 13.0 $21.3 \mathrm{MPa}^{[10,11]}$. 目前的研究表明通过添加助剂、或调变 金属组分的比例、以及优化催化剂的微环境结构等, 可 使反应条件温和很多.

1980 年, 美国联合化学公司的 Grey 和 Pez 研究小 组 ${ }^{[2 a, 12 b]}$ 合成了膦配体稳定的钉氢离子型化合物 1 和 2 (表 1). 他们在探索醛、酮、碳酸酯以及腈类分子催化 加氢反应后, 首次拓展至草酸二甲酯底物, 但是反应活 性并不理想. 以四氢呋喃(THF)为溶剂, 在 $90{ }^{\circ} \mathrm{C}$ 和 620 $\mathrm{kPa}$ 氢压下, 催化剂 1 在 $20 \mathrm{~h}$ 内催化生成 $10 \%$ 的乙醇酸 甲酯(Entry 1), 2 可以催化得到 70\%的产率(Entry 2). 当 反应体系中加入 18-冠醚-6 试图络合稳定钾离子, 提高 钉中心对底物分子的转换频率, 但是结果并不好, 产率 反而降为 $53 \%$ (Entry 3 ). 作者在文中只是提及草酸二甲 酯是一种活性酯, 活性程度可类比于三氟甲基乙酸酯, 但没有讨论反应机理. 不过他们在引言中述及, 对于如 1 和 2 型的催化剂, 反应过程可能经历阴离子部分 $\mathrm{M}-$ $\mathrm{H}$ 键对 $\mathrm{C}=\mathrm{O}$ 键的加成, 再进一步与 $\mathrm{H}_{2}$ 作用并解离转换 形成产物, 活性组分还原. 其中 $\mathrm{MH}_{2}$ 的生成是 $\mathrm{H}_{2}$ 基团 交换的结果还是钓中心氧化加成的结果未有提及, 这一 点实际上对揭示催化剂作用的本质非常重要. A 正离子 对 $\mathrm{C}=\mathrm{O}$ 氢化反应后成盐作用显而易见, 这也可以解释 为什么加入 18-冠醚-6 反而会引起转化率的下降, 因为 18-冠醚-6 络合 A 离子降低了成盐作用(Scheme 4). 尽管 如此, 这种推测的作用方式与上述氢化试剂的 Meerwein- Ponndorf-Verley 反应过程不同 ${ }^{[12 c, 12 \mathrm{~d}]}$.

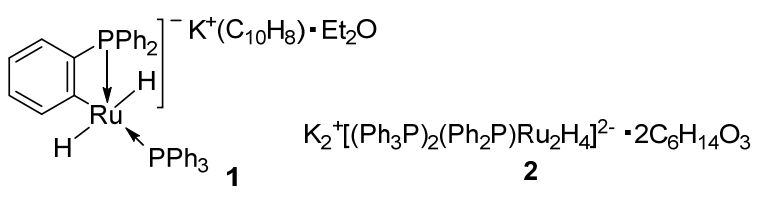

表 1 钉氢化物阴离子催化 $\mathrm{DMO}$ 加氢转化为 $\mathrm{MG}^{a}$ Table 1 Transformation of DMO to MG catalyzed by the ruthenium hydride anion

\begin{tabular}{cccc}
\hline \multirow{2}{*}{ Entry } & \multirow{2}{*}{ Cat. } & \multicolumn{2}{c}{ Yield $/ \%$} \\
\cline { 2 - 4 } & & MG & EG \\
\hline 1 & $\mathbf{2}$ & 10 & 0 \\
2 & $\mathbf{2} / 18$-crown-6 & 50 & 0 \\
3 & ${ }^{a}$ Solve
\end{tabular}

${ }^{a}$ Solvent: THF, $p\left(\mathrm{H}_{2}\right): 620 \mathrm{kPa}$, temperature: $90{ }^{\circ} \mathrm{C}$, time: $20 \mathrm{~h}$.

1984 年, Matteoli 课题组 ${ }^{[13]}$ 也对草酸酯加氢催化进 行了研究. 他们合成了中性钓宸基氢化物 3 和 4 (表 2), 随后考察了催化性能. 在 $180{ }^{\circ} \mathrm{C} 、 13.2 \mathrm{MPa} \mathrm{H}_{2}$ 压、苯 溶剂中, 化合物 3 在 $144 \mathrm{~h}$ 内催化 $51.1 \%$ 草酸二甲酯生 成乙醇酸甲酯(Entry 1); 当催化剂量增加 1 倍时, 反应 在 $39 \mathrm{~h}$ 内完成, 转化率达 $100 \%$ (Entry 2), 但是 4 没有活

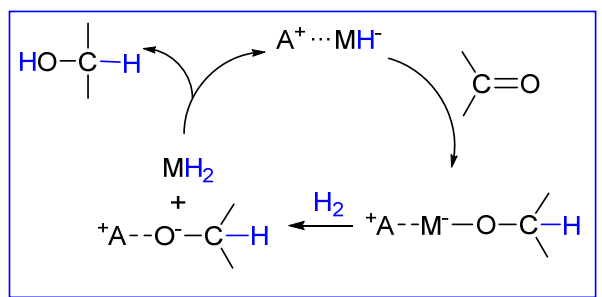

图式 4 Grey和 Pez 建议的金属氢化物离子催化丙酮加氢反应 的可能机理

Scheme 4 Grey and Pez proposed mechanism for hydrogenation of acetone catalyzed by the ruthenium hydride anion

性. 3 和 4 的差别仅是所用膦配体不同，但两者有着如此 大的差异，作者没有深究，也没有探讨相关反应机理. 值得注意的是，该反应条件与负载型催化剂的接近. 于 是他们进一步以碳负载的 $\mathbf{5}$ 为催化剂，在相同条件下考 察表明，反应活性非常低，转化率仅为 3.0\% (Entry 4).

$$
\begin{array}{ccc}
\mathrm{H}_{4} \mathrm{Ru}_{4}(\mathrm{CO})_{8}\left(\mathrm{PBu}_{3}\right)_{4} & \mathrm{H}_{4} \mathrm{Ru}_{4}(\mathrm{CO})_{8}\left(\mathrm{PPh}_{3}\right)_{4} & \mathrm{Ru} / \mathrm{C} \\
\mathbf{3} & \mathbf{4} & \mathbf{5}
\end{array}
$$

表 2 钉氢化物催化 $\mathrm{DMO}$ 加氢转化为 $\mathrm{MG}^{a}$

Table 2 Ruthenium hydride-catalyzed transformation of DMO to $\mathrm{MG}$

\begin{tabular}{ccccc}
\hline \multirow{2}{*}{ Entry } & \multirow{2}{*}{ Cat. } & \multirow{2}{*}{ t/h } & \multicolumn{2}{c}{ Yield/\% } \\
\cline { 4 - 5 } & & & MG & EG \\
\hline 1 & $\mathbf{3}^{b}$ & 144 & 51.1 & 0 \\
2 & $\mathbf{3}^{c}$ & 39 & 100 & 0 \\
3 & $\mathbf{4}$ & 144 & 0.0 & 0 \\
4 & $\mathbf{5}^{d}$ & 144 & 3.0 & 0 \\
\hline
\end{tabular}

${ }^{a}$ Solvent: benzene, $p\left(\mathrm{H}_{2}\right): 13.2 \mathrm{MPa}$, temperature: $180{ }^{\circ} \mathrm{C} ;{ }^{b}$ amount of 0.05 $\mathrm{g} ;{ }^{c}$ amount of $0.10 \mathrm{~g} ;{ }^{d}$ amount of $0.05 \mathrm{~g} / 1 \mathrm{~g}(\mathrm{C})$

在 3 的催化反应中, $\mathrm{H}_{4} \mathrm{Ru}_{4}(\mathrm{CO})_{9}\left(\mathrm{PBu}_{3}\right)_{3}$ 和 $\mathrm{H}_{4} \mathrm{Ru}_{4}-$ $(\mathrm{CO})_{10}\left(\mathrm{PBu}_{3}\right)_{2}$ 物种被检测到, 表明在反应过程中发生了 膦配体的解离，因而促进了低配位活性钓中心的形成. 碳负载金属钓催化剂通过 $\mathrm{H}_{4} \mathrm{Ru}_{4}(\mathrm{CO})_{12}$ 在碳上热分解制 备, 该催化剂显示极低活性, 显然膦配体较碳载体具更 好的配位稳定作用, 有利于活性中心的形成. 催化剂 $\mathbf{3}$ 对草酸二乙酯也显示反应活性，如在 Entry 4 的条件下， 草酸二乙酯的转化率为 $37.7 \%$, 但是产物很复杂.

1985 年, 该研究小组 ${ }^{[15]}$ 报道了系列含膦配体的羰 基钉乙酸化合物以及催化性能研究(表 3). 仍在 13.2 $\mathrm{MPa}$ 氢压、 $180{ }^{\circ} \mathrm{C}$ 、苯溶剂中, 三苯基膦配位的钓化合 物 $\mathbf{1 1}$ 的反应活性与上述结果类似, 仍然极低 $(144 \mathrm{~h}$ 后生 成 $6.0 \%$ 乙醇酸甲酯, Entry 20), 相比较而言, 其它膦配 体羰基钓化合物均显示较好活性. 在该条件下，多核化 合物 6 可以转化 $51.5 \%$ 的草酸二甲酯为乙醇酸甲酯 (Entry 1), 而双核化合物 $\mathbf{1 0}$ 的转化效率为 $41.3 \%$ (Entry 19). 化合物 7 9 的结果随反应条件的变化而变化. 


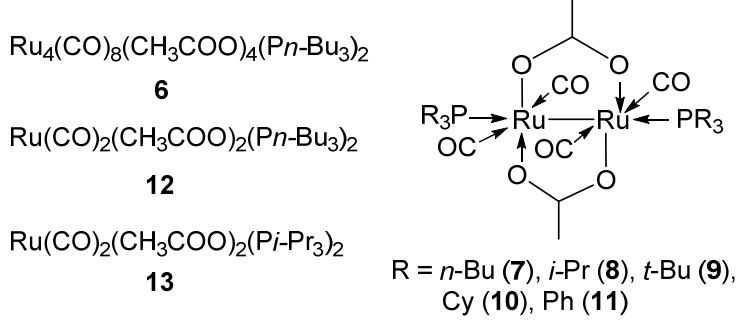

表 3 钉羰基化合物催化 $\mathrm{DMO}$ 加氢转化为 $\mathrm{MG}$ 和 $\mathrm{EG}^{a}$

Table 3 Ruthenium carbonyl-catalyzed transformation of DMO to $\mathrm{MG}$ and $\mathrm{EG}$

\begin{tabular}{ccccccc}
\hline \multirow{2}{*}{ Entry } & \multirow{2}{*}{ Cat. } & \multirow{2}{*}{$*\left(\mathrm{H}_{2}\right) / \mathrm{MPa}$} & \multirow{2}{*}{$T /^{\circ} \mathrm{C}$} & \multirow{2}{*}{$t / \mathrm{h}$} & \multicolumn{2}{c}{ Yield/\% } \\
\cline { 5 - 7 } & & 13.2 & 180 & 144 & 51.5 & 0 \\
$1^{b}$ & $\mathbf{6}$ & 13.2 & 180 & 144 & 79.2 & 0 \\
$2^{b}$ & $\mathbf{7}$ & 13.2 & 180 & 2 & 97.9 & 2.1 \\
3 & $\mathbf{7}$ & 13.2 & 180 & 22 & 13.4 & 0 \\
4 & $\mathbf{7}$ & 13.2 & 180 & 72 & 61.6 & 38.4 \\
5 & $\mathbf{7}$ & 13.2 & 130 & 72 & 96.1 & 3.9 \\
6 & $\mathbf{7}$ & 13.2 & 130 & 2 & 70.1 & 4.3 \\
7 & $\mathbf{8}$ & 13.2 & 180 & 0 \\
8 & $\mathbf{8}$ & 13.2 & 180 & 22 & 71.1 & 0 \\
9 & $\mathbf{8}$ & 13.2 & 180 & 72 & 1.9 & 32.6 \\
10 & $\mathbf{8}$ & 13.2 & 150 & 72 & 15.3 & 21.0 \\
11 & $\mathbf{8}$ & 13.2 & 135 & 72 & 54.1 & 10.2 \\
12 & $\mathbf{8}$ & 13.2 & 120 & 72 & 72.3 & 27.7 \\
13 & $\mathbf{8}$ & 20.3 & 120 & 72 & 40.5 & 59.5 \\
14 & $\mathbf{8}$ & 1.0 & 120 & 72 & 17.2 & 6.1 \\
15 & $\mathbf{9}$ & 13.2 & 180 & 72 & 7.7 & 11.7 \\
16 & $\mathbf{9}$ & 13.2 & 180 & 2 & 66.3 & 0.8 \\
17 & $\mathbf{9}$ & 13.2 & 180 & 22 & 29.2 & 0 \\
18 & $\mathbf{9}$ & 13.2 & 120 & 72 & 81.9 & 18.1 \\
$19^{b}$ & $\mathbf{1 0}$ & 13.2 & 180 & 144 & 41.3 & 0 \\
$20^{b}$ & $\mathbf{1 1}$ & 13.2 & 180 & 144 & 6.0 & 0 \\
21 & $\mathbf{1 2}$ & 13.2 & 180 & 2.50 & 100 & 0 \\
22 & $\mathbf{1 3}$ & 13.2 & 180 & 72 & 28.7 & 66.5 \\
\hline
\end{tabular}

${ }^{a}$ Solvent: $\mathrm{MeOH} ;{ }^{b}$ benzene.

针对催化剂 7, 在保持 $13.2 \mathrm{MPa}$ 氢压和 $180{ }^{\circ} \mathrm{C}$ 下苯 (144 h 后乙醇酸甲酯的得率为 $79.2 \%$, Entry 2 ) 和甲醇溶 剂( $22 \mathrm{~h}$ 后的得率为 $13.4 \%$, Entry 4) 对反应有很大影响; 进一步以甲醇为溶剂, 温度和反应时间对转化率也显示 影响 $\left(120{ }^{\circ} \mathrm{C} 、 72 \mathrm{~h}\right.$ 后生成 $96.1 \%$ 的乙醇酸甲酯和 $3.9 \%$ 的乙二醇, Entry $6 ; 180{ }^{\circ} \mathrm{C} 、 2 \mathrm{~h}$ 时生成 $97.9 \%$ 的乙醇酸 甲酯和 $2.1 \%$ 的乙二醇, Entry 3, $72 \mathrm{~h}$ 后生成 $61.8 \%$ 的乙醇 酸甲酯和 $31.4 \%$ 的乙二醇, Entry 5). 这些数据表明乙醇 酸甲酯主要是低温下的反应产物, 升高温度则促进乙二 醇的生成，但同时导致产物分解，如生成甲酸甲酯、二 氧化碳和甲烷等. 因此, 高温长时间内测得的数据实际 上是产物分解后的数据. 催化剂 8 和 9 的结果与 $\mathbf{7}$ 相比 具体数值有一定差异, 但受温度和时间的影响类似, 其 中 8 的结果也同时受到 $\mathrm{H}_{2}$ 压的影响.

单核化合物 12 和 13 也具有催化活性(表 3). 以 12
为催化剂，控制 $13.2 \mathrm{MPa}$ 氢气压和 $180{ }^{\circ} \mathrm{C}$ 反应温度，在 甲醇中草酸二甲酯氢化时 $0 \sim 2.5 \mathrm{~h}$ 内几乎只得到乙醇酸 甲酯和甲醇, 反应呈 0.5 级(在苯溶剂中呈 1 级反应特 征); 延长反应时间，另一种产物乙二醇逐步生成，但是 不具有任何动力学转化的特征. 一方面催化剂的活性逐 渐降低, 反应转化率也随之降低; 另一方面出现其它副 产物如二氧化碳、乙酸甲酯、二(2-差列乙基)醚和乙醇酸2-羟基乙酯等.

1991 年, 他们 $^{[15]}$ 报道 $\mathbf{1 3}$ 可将草酸二甲酯选择性地 还原为乙二醇. 为避免底物的分解, 氢化反应需要在 $120{ }^{\circ} \mathrm{C}$ 下进行, 促进草酸二酯完全转化, 之后再在 $180{ }^{\circ} \mathrm{C}$ 下将中间体乙醇酸甲酯氢化.

总的来说, 催化剂 6 13 对产物的选择性受温度控 制，但是过高温度引起产物的分解; 同时可以看出不同 膦配体的催化剂结果有差异. 具体的催化反应机理未有 讨论.

1997 年, Elsevier 课题组 ${ }^{[16]}$ 选择乙酰丙酮钉 $\left(\mathrm{Ru}(\mathrm{acac})_{3}\right)$ 作为金属前驱体，与系列配体组成催化剂体 系考察了草酸二甲酯的加氢反应. Hara 和 Wada 课题 组 ${ }^{[17]}$ 已经研究了乙酰丙酮钉/配体催化酸酤氢化为內酯 的反应，反应在甲醇中进行，同时需要金属锌的参与. 他们认为锌促进乙酰丙酮钓的快速还原，原位产生催化 活性中心(表 4). 在 $100{ }^{\circ} \mathrm{C} 、 7.1 \mathrm{MPa}$ 氢压、甲醇溶剂中, 单独使用乙酰丙酮钉/锌基本上没有活性(41 h, $2 \% \mathrm{MG}$, Entry 1); 加入配体 $\mathrm{AsPh}_{3} 、 \mathrm{PCy}_{3}$ 、邻菲罗啉双氮 L21、 三氮 L22 以及吡唑硼氢钾盐 $\mathbf{L 2 3}$ 也均无活性. 相比较而 言，其它单齿、双齿和多齿配体的加入能够催化反应的 进行. 以 $16 \mathrm{~h}$ 为计量时间, 加入 $\mathrm{PPh}_{3} 、 \mathbf{L 1 7} 、 \mathbf{L 1 8} 、 \mathbf{L 1 9}$ 分别生成 $36 \% 、 11 \% 、 67 \% 、 85 \%$ 的乙醇酸甲酯(Entries 2 和 5 7), 而加入三脚架型膦配体 $\mathbf{L 2 0}$ 会同时生成乙醇 酸甲酯和乙二醇，两者的量随锌使用量变化，在该反应 条件下最好的结果是锌的加入量为 $20 \%$ 时，草酸二甲酯 的转化率达 $100 \%$, 乙二醇的生成量为 $95 \%$ (Entry 8 ).

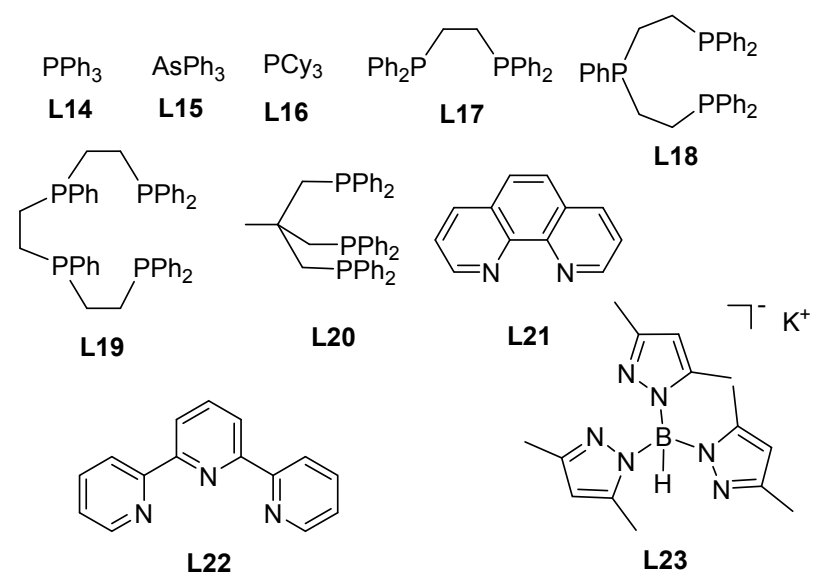


表 4 不同配体一钉化合物催化 DMO 加氢转化为 $\mathrm{MG}$ 和 $\mathrm{EG}$ 的反应结果 ${ }^{a}$

Table 4 Results for hydrogenation of DMO to MG and EG catalyzed by $\mathrm{Ru}$ complexes incorporating varied ligands

\begin{tabular}{|c|c|c|c|c|c|c|}
\hline \multirow{2}{*}{ Entry } & \multirow{2}{*}{ Cat. } & \multirow{2}{*}{$p\left(\mathrm{H}_{2}\right) / \mathrm{MPa}$} & \multirow{2}{*}{$T /{ }^{\circ} \mathrm{C}$} & \multirow{2}{*}{$t / \mathrm{h}$} & \multicolumn{2}{|c|}{ Yield/\% } \\
\hline & & & & & MG & EG \\
\hline 1 & $\mathrm{Ru}(\mathrm{acac})_{3} / \mathrm{Zn}$ & 7.0 & 100 & 41 & 2 & 0 \\
\hline 2 & $\mathrm{Ru}(\mathrm{acac})_{3} / \mathbf{L 1 4} / \mathrm{Zn}$ & 7.0 & 100 & 16 & 36 & 0 \\
\hline 3 & $\mathrm{Ru}(\mathrm{acac})_{3} / \mathbf{L 1 5} / \mathrm{Zn}$ & 7.0 & 100 & 16 & 0 & 0 \\
\hline 4 & $\mathrm{Ru}(\mathrm{acac})_{3} / \mathbf{L 1 6} / \mathrm{Zn}$ & 7.0 & 100 & 16 & 1 & 0 \\
\hline 5 & $\mathrm{Ru}(\mathrm{acac})_{3} / \mathbf{L 1 7} / \mathrm{Zn}$ & 7.0 & 100 & 16 & 11 & 0 \\
\hline 6 & $\mathrm{Ru}(\mathrm{acac})_{3} / \mathbf{L 1 8} / \mathrm{Zn}$ & 7.0 & 100 & 16 & 67 & 0 \\
\hline 7 & $\mathrm{Ru}(\mathrm{acac})_{3} / \mathbf{L 1 9} / \mathrm{Zn}$ & 7.0 & 100 & 16 & 85 & 0 \\
\hline 8 & $\mathrm{Ru}(\mathrm{acac})_{3} / \mathbf{L 2 0} / \mathrm{Zn}^{b}$ & 7.0 & 100 & 16 & 1 & 95 \\
\hline 9 & $\mathrm{Ru}(\mathrm{acac})_{3} / \mathbf{L 2 0} / \mathrm{Zn}^{c}$ & 7.0 & 100 & 16 & 15 & 52 \\
\hline 101 & $\mathrm{Ru}(\mathrm{acac})_{3} / \mathbf{L 2 0} / \mathrm{Zn}$ & 3.8 & 100 & 16 & 24 & 10 \\
\hline 111 & $\mathrm{Ru}(\mathrm{acac})_{3} / \mathbf{L 2 0} / \mathrm{Zn}$ & 2.0 & 100 & 16 & 19 & 2 \\
\hline 121 & $\mathrm{Ru}(\mathrm{acac})_{3} / \mathbf{L 2 0}$ & 7.0 & 100 & 16 & 24 & 22 \\
\hline 131 & $\mathrm{Ru}(\mathrm{acac})_{3} / \mathbf{L 2 0} / \mathrm{Zn}^{d}$ & 2.0 & 100 & 16 & 0 & 84 \\
\hline $14 \mathrm{I}$ & $\mathrm{Ru}(\mathrm{acac})_{3} / \mathbf{L 2 0}^{\mathrm{c}}$ & 2.0 & 100 & 16 & 10 & 63 \\
\hline $15 \mathrm{I}$ & $\mathrm{Ru}(\mathrm{acac})_{3} / \mathbf{L} \mathbf{2 1} / \mathrm{Zn}$ & 7.0 & 100 & 16 & 0 & 0 \\
\hline $16 \mathrm{I}$ & $\mathrm{Ru}(\mathrm{acac})_{3} / \mathbf{L 2 2} / \mathrm{Zn}$ & 7.0 & 100 & 16 & 0 & 0 \\
\hline $17 \mathrm{I}$ & $\mathrm{Ru}(\mathrm{acac})_{3} / \mathbf{L 2 3} / \mathrm{Zn}$ & 7.0 & 100 & 16 & 1 & 0 \\
\hline
\end{tabular}

${ }^{a}$ Solvent: $\mathrm{MeOH} ;{ }^{b} 22 \% \mathrm{Zn} ;{ }^{c} 24 \% \mathrm{Zn} ;{ }^{d}$ in dry $\mathrm{MeOH}$.

明显地可以看出, 配体对金属钓的络合稳定对催化 活性起着非常重要的作用, 膦配体的稳定作用要远好于 氮配体; 在膦配体中稳定作用顺序大致为: $\mathrm{PCy}_{3}<$ $\mathrm{Ph}_{2} \mathrm{PC}_{2} \mathrm{H}_{4} \mathrm{PPh}_{2}<\mathrm{PPh}_{3}<\mathrm{PhP}\left(\mathrm{C}_{2} \mathrm{H}_{4} \mathrm{PPh}_{2}\right)_{2} \approx\left[\mathrm{CH}_{2} \mathrm{P}(\mathrm{Ph})-\right.$ $\left.\mathrm{C}_{2} \mathrm{H}_{4} \mathrm{PPh}_{2}\right]_{2} \ll \mathrm{MeC}\left(\mathrm{CH}_{2} \mathrm{PPh}_{2}\right)$.

相比于 Matteoli 课题组的工作, Elsevier 课题组的催 化剂体系活性高, 且反应条件温和, 其中关键是多齿膦 配体的使用. $\mathrm{MeC}\left(\mathrm{CH}_{2} \mathrm{PPh}_{2}\right)_{3}$ 配体能够与钓中心形成“口 袋式” 配位, 给钉中心与底物分子间的作用提供了所要 求的电子和立体空间, 这一点非常必要. 基于温和的反 应条件, 副反应以及产物分解反应也得到了一定程度的 遏制.

对 $\mathrm{Ru}(\mathrm{acac})_{3} / \mathbf{L 2 0} / \mathrm{Zn}$ 体系, 不论提升压力还是增加 催化剂浓度均能提高草酸二甲酯的转化率(单位时间的 转换频率也增加), 但是 $\mathrm{Zn}$ 的加入并不是决定因素(无 $\mathrm{Zn}$ 参与仍显示催化活性, 见 Entries 12 和 14), 在相同条 件下加入 $Z n$ 总体上会提高反应活性.

2003 年, 他们 ${ }^{[18]}$ 将工作延伸至其它金属前驱体, 发 现使用 $\mathrm{RuCl}_{2}\left(\mathrm{PPh}_{3}\right)_{4}$, 在 $120{ }^{\circ} \mathrm{C} 、 8.1 \mathrm{MPa}$ 氢压下甲醇溶 剂中 $17 \mathrm{~h}$ 内, 草酸二甲酯的转化率为 $51 \%$, 乙醇酸甲酯 的收率仅为 $20 \%$, 没有乙二醇生成; 而在 THF 溶剂中草 酸二甲酯的转化率为 $37 \%$ 和乙醇酸甲酯的收率为 $12 \%$. 原位混合的 $\mathrm{RuCl}_{3} / \mathrm{PPh}_{3}$ 给出 $44 \%$ 的转化率和 $15 \%$ 的收 率, 同样没有乙二醇产生. 以 $\mathrm{RuCl}_{2}\left(\mathrm{PPh}_{3}\right)_{4}$ 为前驱体的 体系如何转化生成活性氢化物物种至今尚不明确.

2006 年, Hanton 研究小组 ${ }^{[19]}$ 报道了一种三脚架型含
硫配体 $\mathrm{MeC}\left(\mathrm{CH}_{2} \mathrm{SBu}\right)_{3}(\mathbf{L 2 7})$, 并研究了与 $\mathrm{Ru}(\mathrm{acac})_{3}$ 组 成的催化剂体系用于草酸二甲酯的选择性氢化. 含硫配 体已经被报道与 Rh、Pd、 Ir 和 Pt 形成络合物, 用于烯 烃和酮类分子的加氢催化，但是从未用于酯类分子 ${ }^{[20]}$. 在 $100{ }^{\circ} \mathrm{C} 、 8.0 \mathrm{MPa}$ 氢压、甲醇溶剂中, Hanton 研究小 组考察证实没有膦配体参与的 $\mathrm{Ru}(\mathrm{acac})_{3}$ 无活性, 该结 果印证了 Elsevier 组的考察结果(表 5). 因而加入 POct $_{3}$ 反应具有活性, 但需要 $304 \mathrm{~h}$ 完成草酸二甲酯完全转化 为乙醇酸甲酯(Entry 2), 加入 $\mathrm{PPh}_{3}$ 在 $5.7 \mathrm{~h}$ 后全部转化为 乙二醇(Entry 1). 尽管反应条件中 $\mathrm{H}_{2}$ 压微有差异, 但是 后者的结果与 Elsevier 组的相差很大(表 5 Entry $1 \mathrm{vs}$ 表 4 Entry 2). 当使用硫环配体时, L25 和 L26 没有促进作 用, 而三脚架型 $\mathbf{L 3 1}$ 显示很好的作用, 随着反应时间从 $23 \mathrm{~h}$ 增加到 $136 \mathrm{~h}$, 乙醇酸甲酯的收率从 $36.5 \%$ 增加到 $100 \%$ (表 5 Entries 5 7), 但没有乙二醇生成. 在前两个 反应中，可以观察到 “钓黑” 的生成，表明 L25 和 $\mathbf{L 2 6}$ 并不能有效地配位稳定钓中心，导致钓被还原为金属

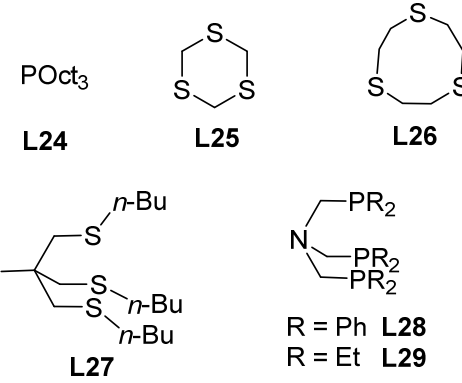

表 5 不同配体-钉化合物催化 DMO 加氢转化为 $\mathrm{MG}$ 和 $\mathrm{EG}$ 的反应结果

Table 5 Results for hydrogenation of DMO to MG and EG catalyzed by $\mathrm{Ru}$ complexes incorporating varied ligands

\begin{tabular}{ccccccc}
\hline \multirow{2}{*}{ Entry } & \multirow{2}{*}{ Cat. } & \multirow{2}{*}{$p\left(\mathrm{H}_{2}\right) / \mathrm{MPa}$} & \multirow{2}{*}{${ }^{\circ} \mathrm{C}$} & \multirow{2}{*}{$t / \mathrm{h}$} & \multicolumn{3}{c}{ Yield/\% } \\
\cline { 5 - 7 } & & & & & $\mathrm{MG}$ & $\mathrm{EG}$ \\
\hline 1 & $\mathrm{Ru}(\mathrm{acac})_{3} / \mathbf{L 1 8} / \mathrm{Zn}$ & 8.0 & 100 & 5.7 & 0 & 100 \\
2 & $\mathrm{Ru}(\mathrm{acac})_{3} / \mathbf{L 2 4} / \mathrm{Zn}$ & 8.0 & 100 & 304 & 100 & 0 \\
3 & $\mathrm{Ru}(\mathrm{acac})_{3} / \mathbf{L 2 5} / \mathrm{Zn}$ & 8.0 & 100 & 20 & 0 & 0 \\
4 & $\mathrm{Ru}(\mathrm{acac})_{3} / \mathbf{L 2 6} / \mathrm{Zn}$ & 8.0 & 100 & 20 & 0 & 0 \\
5 & $\mathrm{Ru}(\mathrm{acac})_{3} / \mathbf{L 2 7} / \mathrm{Zn}$ & 8.0 & 100 & 23 & 36.9 & 0 \\
6 & $\mathrm{Ru}(\mathrm{acac})_{3} / \mathbf{L 2 7} / \mathrm{Zn}$ & 8.0 & 100 & 69 & 87.2 & 0 \\
7 & $\mathrm{Ru}(\mathrm{acac})_{3} / \mathbf{L 2 7} / \mathrm{Zn}$ & 8.0 & 100 & 136 & 100 & 0 \\
8 & $\mathrm{Ru}(\mathrm{acac})_{3} / \mathbf{L 2 8} / \mathrm{Zn}$ & 8.0 & 100 & 20.9 & 96.6 & 0 \\
9 & $\mathrm{Ru}(\mathrm{acac})_{3} / \mathbf{L 2 8} / \mathrm{Zn}$ & 6.0 & 100 & 66 & 35.9 & 64.1 \\
10 & $\mathrm{Ru}(\mathrm{acac})_{3} / \mathbf{L 2 8} / \mathrm{Zn}$ & 7.0 & 100 & 84 & 58.2 & 41.8 \\
11 & $\mathrm{Ru}(\mathrm{acac})_{3} / \mathbf{L 2 8} / \mathrm{Zn}$ & 8.0 & 100 & 66 & 46.5 & 53.5 \\
12 & $\mathrm{Ru}(\mathrm{acac})_{3} / \mathbf{L 2 8} / \mathrm{Zn}$ & 11.0 & 100 & 34 & 97.3 & 2.7 \\
13 & $\mathrm{Ru}(\mathrm{acac})_{3} / \mathbf{L 2 8} / \mathrm{Zn}$ & 14.0 & 100 & 86 & 80.2 & 19.8 \\
14 & $\mathrm{Ru}(\mathrm{acac})_{3} / \mathbf{L 2 8} / \mathrm{Zn}$ & 8.0 & 80 & 82 & 80.9 & 18.1 \\
15 & $\mathrm{Ru}(\mathrm{acac})_{3} / \mathbf{L 2 8} / \mathrm{Zn}$ & 8.0 & 90 & 61 & 46.9 & 53.1 \\
16 & $\mathrm{Ru}(\mathrm{acac})_{3} / \mathbf{L 2 8} / \mathrm{Zn}$ & 8.0 & 110 & 64 & 60.0 & 40.0 \\
17 & $\mathrm{Ru}(\mathrm{acac})_{3} / \mathbf{L 2 8} / \mathrm{Zn}$ & 8.0 & 120 & 41 & 58.2 & 41.8 \\
18 & $\mathrm{Ru}(\mathrm{acac})_{3} / \mathbf{L 2 9} / \mathrm{Zn}$ & 8.0 & 100 & 20.6 & 93.0 & 5.6 \\
\hline${ }^{a} \mathrm{Solvent:} \mathrm{MeOH}$. & & & & &
\end{tabular}


态. 在使用 $\mathbf{L 2 7}$ 的所有反应中, 打开高压釜仍然观察到 黄色透明溶液, 没有任何沉淀. 这说明三脚架型硫配体 在这些条件下具有超强的络合稳定 $\mathrm{Ru}(\mathrm{II})$ 的能力.

添加和不添加 $\mathrm{Zn}$ 的催化体系均需要一段时间才能 观察到反应的发生, 这预示着 $\mathrm{Ru}(\mathrm{III})$ 前驱体在反应中转 化为 $\mathrm{Ru}(\mathrm{II})$ 活性物种需要时间, 即存在一个诱导期, 锌 的添加的确有助于缩短诱导期, 因而反应速率得到提 高. 相比于三脚架型膦配体, 三脚架型硫配体只能将草 酸二甲酯还原为乙醇酸甲酯, 且与反应时间无关. 在新 鲜配制的催化剂溶液中直接加入乙醇酸甲酯底物, 也没 有任何转化. 由此可见不同配体影响着钓的催化能力. 进一步研究发现 $\mathrm{Ru}(\mathrm{acac})_{3} / \mathrm{L27} / \mathrm{Zn}$ 和 $\mathrm{Ru}(\mathrm{acac})_{3} / \mathbf{L 1 8} / \mathrm{Zn}$ 体系体系对底物分子都呈零级反应, 说明草酸二甲酯的 浓度对反应无影响.

2011 年, Hanton 研究小组 ${ }^{[21]}$ 考察了 $\mathrm{Ru}(\mathrm{acac})_{3} / \mathbf{L 1 8}$ 体系添加 Zn 以及其它有机化合物的影响(图 1). 他们首 先证实该反应体系存在诱导期, 即将催化组分和底物混 合后需要一段时间才观察到反应的进行. Zn 的加入可以 将反应诱导期从 $160 \mathrm{~min}$ 缩短到 $30 \mathrm{~min}$ ，同时反应时间 也会从 $10.1 \mathrm{~h}$ 减少到 $5.7 \mathrm{~h}$. 但是加入如图 1 所示的有机 分子添加剂(30，1,5,7-三氮杂二环 [4.4.0]-5-癸烯; DAE， 二异丙基氨基乙醇; DMAP, 4-二甲氨基吡啶; 4NP, 4-硝 基苯酚)，诱导期时间会缩短，不过反应时间均会拉长， 同时产物在数和量上也会变化. 对吡啶、L30、DMAP 添加剂, 仅生成产物乙醇酸甲酯; 对苯酚, 得到乙二醇; 而其它添加剂则会导致生成乙醇酸甲酯和乙二醇的混 合物，且百分占比不同. 显然这些有机物添加剂对草酸 二甲酯氢化速率并没有起到促进作用. 推测这些添加剂 有可能以两种不同的方式作用，一种是通过电子给体的 作用协助催化活性物种的生成; 另一种则是与底物作 用. 推测当后者起主导作用时, 大大影响了催化反应速 率.

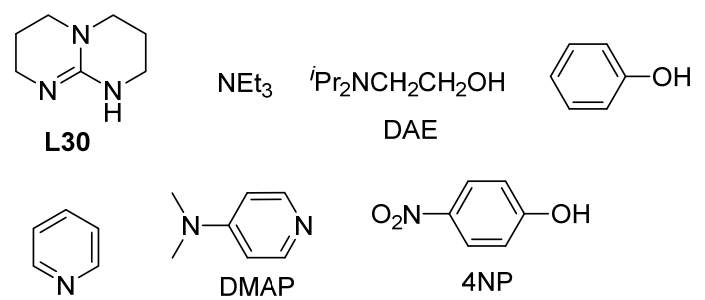

图 1 Hanton 组用于 $\mathrm{Ru}(\mathrm{acac})_{3} / \mathbf{L 1 8}$ 体系的有机添加剂 Figure 1 Additives added in $\mathrm{Ru}(\mathrm{acac})_{3} / \mathbf{L 1 8}$ system reported by Hanton group

在酯的氢化反应中, 工业界特别关注的是催化体系 对有机羒酸杂质的耐受度, 许多多相催化技术往往受制 于这些杂质, 因为它们会引起催化剂的流失. 为此, Hanton 研究组研究了 $\mathrm{Ru}(\mathrm{acac})_{3} / \mathbf{L 1 8} / \mathrm{Zn}$ 体系受羧酸的影
响. 他们在加入草酸二甲酯的同时也加入 $10 \mathrm{~mol} \%$ 的乙 醇酸, 在 $100{ }^{\circ} \mathrm{C} 、 8.0 \mathrm{MPa}$ 氢压、甲醇溶剂中, 经过 60 $\min$ 的诱导期和 $12 \mathrm{~h}$ 的反应时间, $100 \%$ 的反应物转化为 乙二醇. GC-MS 没有检测到乙醇酸. 其它实验也表明乙 醇酸在甲醇中 $60{ }^{\circ} \mathrm{C}$ 下完全转化为乙醇酸甲酯, 因此加 入的乙醇酸应该先酯化然后被氢化, 再转化为乙二醇.

强 $\sigma$-电子配位的膦配体是很适合的稳定钓金属的 配体，呈多齿配位特性的配体则更佳. 配体 L27 和 L28 有类似于 $\mathbf{L 2 0}$ 的三脚架型的配位特征，同时 $\mathrm{N}$ 原子也会 参与金属的配位，因此这两个配体配位的钓化合物是很 好的酯加氢催化剂. Hanton 组证实在 $100{ }^{\circ} \mathrm{C} 、 8.0 \mathrm{MPa}$ 氢压、甲醇溶剂中, 在 $20 \mathrm{~h}$ 内 $\mathrm{Ru}(\mathrm{acac})_{3} / \mathbf{L 2 7} / \mathrm{Zn}$ 催化草 酸二甲酯生成 $96.6 \%$ 的乙醇酸甲酯(表 5 Entry 8 ), 而 $\mathrm{Ru}(\mathrm{acac})_{3} / \mathbf{L 2 8} / \mathrm{Zn}$ 催化生成 $93.0 \%$ 的乙醇酸甲酯和 $5.6 \%$ 的乙二醇(表 5 Entry 18). 动力学考察表明这两个反应都 对底物倾向于一级，即反应速率受草酸二甲酯的浓度影 响. 推测在反应过程中草酸二甲酯与钌中心发生强的络 合，该络合步骤是反应的决速步. 尽管氢压的变化对产 物乙醇酸甲酯和乙二醇的生成比例有影响(较低氢压对 生成乙二醇有利而高压对乙醇酸甲酯有利(表 5 Entries 9 13), 但是对反应速率影响甚微, 显然 $\mathrm{H}_{2}$ 分子在钓中 心作用形成金属氢活性物种并不包含在决速步内. 该推 测与动力学观察到的对草酸二甲酯为一级反应的结果 相符. 温度变化对反应影响似乎不大, 在 $80{ }^{\circ} \mathrm{C}$ 下反应 十分缓慢, 以 $10{ }^{\circ} \mathrm{C}$ 为步长从 $90{ }^{\circ} \mathrm{C}$ 升温至 $120{ }^{\circ} \mathrm{C}$, 反 应速率没有明显变化. 普遍认为催化剂会随着温度升高 而加剧 $\mathrm{H}_{2}$ 的氧化加成形成活性物种，同时也促进对酯 羰基的加成，当然也会容易引起催化剂的分解. 很可能 是升高温度带来的速率提升与催化剂分解导致催化剂 浓度降低而引起的速率降低相抵消，使得总反应表观速 率变化不大.

依据上述系列结果，他们提出一种可能的反应机 理. 如 Scheme 5 所示, $\mathrm{Ru}(\mathrm{acac})_{3} / \mathrm{L} 27 / \mathrm{Zn}$ 经过诱导期反 应, 脱除戊二醇生成三脚架型膦配体配位的 $\mathrm{Ru}^{\mathrm{II}} \mathrm{H}_{2}$ 物 种. $\mathrm{GC}$ 谱分析到痕量的 2,4-戊二醇予以证实. 这是一个 钓中心 16 电子结构的化合物，易于与底物草酸二甲酯 络合配位. $\mathrm{PPh}_{3}(\mathbf{L 1 8})$ 配体体系观察到对底物呈零级，表 明钉与草酸二甲酯结合与解离都很容易. 然而 L27 配体 体系对底物呈一级，显然由 L27 配位的钓络合草酸二甲 酯容易而解离难，该络合反应是决速步。钓氢插入羰基 后容易形成 $\mathrm{RuCO}_{2}$ 四元环结构, 再经还原消除生成半 缩醛分子和 $[\mathbf{L 2 7}] \mathrm{Ru}(0)$ 物种, $[\mathbf{L 2 7}] \mathrm{Ru}(0)$ 与 $\mathrm{H}_{2}$ 氧化加成 重新还原为 $[\mathbf{L} 27] \mathrm{Ru}^{\mathrm{II}} \mathrm{H}_{2}$ 用于下一个催化反应，半缩醛 分子则自发重排为乙醛酸甲酯，在类似的反应过程中被 氢化为乙醇酸甲酯. 乙二醇分子则是由乙醇酸甲酯经过 
相应的催化循环反应生成.

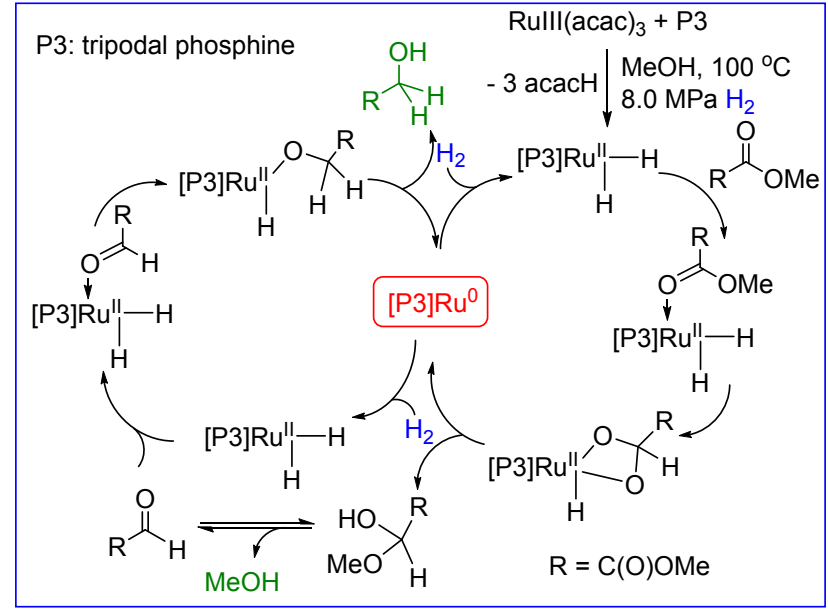

图式 5 Hanton 推测的反应机理

Scheme 5 Mechanism suggested by Hanton group

2013 年, Beller 课题组 ${ }^{[22]}$ 考察了如表 6 所示的系列 催化剂对草酸酯加氢的催化性能. 他们首先使用 Takasago 公司首创的钳型 Macho 钉催化剂 31 ${ }^{[23]}$. 在 $100{ }^{\circ} \mathrm{C} 、 6.0 \mathrm{MPa} \mathrm{H}_{2}$ 、四氢呋喃溶剂和 $\mathrm{NaOEt}$ 存在下, 该 催化剂在 $20 \mathrm{~h}$ 内催化草酸二乙酯直接转化为乙二醇, 收 率为 $92 \%$ (Entry 1). 在相同条件下使用氯离子被氢负离 子 $\mathrm{BH}_{3}$ 加合物替代的催化剂 32 , 给出略高的转化率 (96\%, Entry 2); 控制时间 $1 \mathrm{~h}$, 反应生成 $21 \%$ 的乙醇酸 乙酯和 $72 \%$ 的乙二醇(Entry 3). 该结果说明反应经过乙 醇酸乙酯中间体, 在较长的反应时间内其完全转化为乙 二醇.

在不使用 $\mathrm{NaOEt}$ 的情况下, 32 也具有很好的催化活 性, 在 $1 \mathrm{~h}$ 内转化草酸二乙酯生成 $15 \%$ 的乙醇酸乙酯和 $85 \%$ 的乙二醇(Entry 4 ), $4 \mathrm{~h}$ 后得到大于 $99 \%$ 的乙二醇 (Entry 5). 当降至 $60{ }^{\circ} \mathrm{C}$ 时, $1 \mathrm{~h}$ 内生成 $99 \%$ 的乙醇酸乙 酯(Entry 6); 而室温下 $120 \mathrm{~h}$ 内给出 $7 \%$ 的乙醇酸乙酯和 $86 \%$ 的乙二醇(Entry 7). 同样氢压和溶剂变换也对催化 反应有影响, 在 $60{ }^{\circ} \mathrm{C} 、 3.0 \mathrm{MPa} \mathrm{H}_{2}$ 、四氢呋喃溶剂中 1 $\mathrm{h}$ 下仅生成 $46 \%$ 的乙醇酸乙酯(Entry 8$)$; 而在乙醇溶剂 中、 $60{ }^{\circ} \mathrm{C} 、 6.0 \mathrm{MPa} \mathrm{H}$ 、 $0.5 \mathrm{~h}$ 内生成 $98 \%$ 的乙醇酸乙 酯(Entry 9); 但是使用甲苯或碳酸丙烯酯溶剂, 没有活 性(Entries 10 和 11). 无溶剂参与时, 在 $120{ }^{\circ} \mathrm{C} 、 7.0 \mathrm{MPa}$ $\mathrm{H}_{2} 、 140 \mathrm{~h}$ 内只能产生 $81 \%$ 的乙醇酸乙酯(Entry 12 ).

使用 $\mathrm{NaOEt}$ 可以促进钉中心的卤素基团转化为钉 烷氧基, 进而转化为氢负离子活性基团, 这一点已为很 多课题组的研究证实. 因此, 上述结果表明 32 可以看成 是真正的催化活性物种.

变换 31 和 32 中的 $\mathrm{PPh}_{2}$ 为 $\mathrm{P}^{i} \mathrm{Pr}_{2}$ (催化剂 33 和 34), 相 应的反应速率变化不大，但是只生成乙醇酸乙酯 (Entries 13 和 14). 可以注意到催化剂 31 34 中的配体

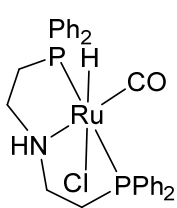

31<smiles>Pc1ccccc1P</smiles>

L35

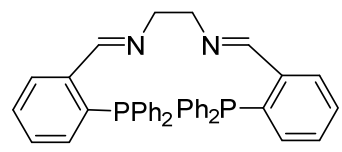

L38

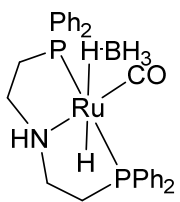

32

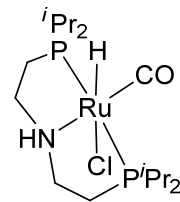

33

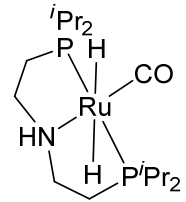

34<smiles>Pc1ccccc1</smiles>

L36

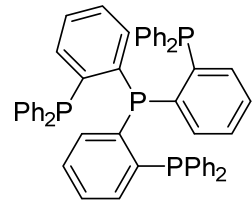

L37
表 6 不同催化体系催化 DMO 加氢转化为乙醇酸乙酯和 $\mathrm{EG}$ 的反应结果

Table 6 Results for hydrogenation of DMO to ethyl glycolate and EG with different catalyst systems

\begin{tabular}{|c|c|c|c|c|c|c|}
\hline \multirow[b]{2}{*}{ Entry } & \multirow[b]{2}{*}{ Cat. } & \multirow[b]{2}{*}{$\begin{array}{c}p\left(\mathrm{H}_{2}\right) / \\
\mathrm{MPa}\end{array}$} & \multirow[b]{2}{*}{$T /{ }^{\circ} \mathrm{C}$} & \multirow[b]{2}{*}{$t / \mathrm{h}$} & \multicolumn{2}{|c|}{ Yield/\% } \\
\hline & & & & & $\begin{array}{c}\text { Ethyl } \\
\text { glycolate }\end{array}$ & EG \\
\hline 1 & 31/ $\mathrm{NaOEt}$ & 6.0 & 100 & 20 & 0 & 92 \\
\hline 2 & 32 $/ \mathrm{NaOEt}$ & 6.0 & 100 & 20 & 0 & 96 \\
\hline 3 & 32/ $\mathrm{NaOEt}$ & 6.0 & 100 & 1 & 21 & 72 \\
\hline 4 & 32 & 6.0 & 100 & 1 & 15 & 85 \\
\hline 5 & 32 & 6.0 & 100 & 4 & 0 & $>99$ \\
\hline 6 & 32 & 6.0 & 60 & 1 & 99 & 0 \\
\hline 7 & 32 & 6.0 & r.t. & 120 & 7 & 86 \\
\hline 8 & 32 & 3.0 & 60 & 1 & 46 & 0 \\
\hline $9^{b}$ & 32 & 6.0 & 60 & 0.5 & 98 & 0 \\
\hline $10^{c}$ & 32 & 9.0 & 60 & 1 & 0 & 0 \\
\hline $11^{d}$ & 32 & 9.0 & 60 & 1 & 0 & 0 \\
\hline $12^{e}$ & 32 & 7.0 & 120 & 140 & 81 & 0 \\
\hline 13 & 33/NaOEt & 6.0 & 100 & 20 & 89 & 0 \\
\hline 14 & 34 $/ \mathrm{NaOEt}$ & 6.0 & 100 & 20 & 96 & 0 \\
\hline 15 & $\mathbf{L 3 5} / \mathrm{Ru}(\mathrm{acac})_{3} / \mathrm{NaOEt}$ & 6.0 & 100 & 20 & 0 & 0 \\
\hline 16 & $\mathbf{L 3 6} / \mathrm{Fe}\left(\mathrm{BF}_{4}\right)_{2} / \mathrm{NaOEt}$ & 6.0 & 100 & 20 & 0 & 0 \\
\hline 17 & L36/Co $\left(\mathrm{BF}_{4}\right)_{2} / \mathrm{NaOEt}$ & 6.0 & 100 & 20 & 0 & 0 \\
\hline 18 & $\mathbf{L 3 7} / \mathrm{Ru}(\mathrm{acac})_{3} / \mathrm{NaOEt}$ & 6.0 & 100 & 20 & 0 & 0 \\
\hline 19 & $\{[\mathbf{L 3 7}] \mathrm{FeF}\} \mathrm{BF}_{4} / \mathrm{NaOEt}$ & 6.0 & 100 & 20 & 0 & 0 \\
\hline 20 & $\mathbf{L 3 8} /\left[\mathrm{Ru}(\mathrm{acac})_{3}\right] / \mathrm{NaOEt}$ & 6.0 & 100 & 20 & 0 & 0 \\
\hline $21^{f}$ & $\mathbf{L 2 0} / \mathrm{Ru}(\mathrm{acac})_{3} / \mathrm{NaOEt}$ & 6.0 & 100 & 20 & 0 & 96 \\
\hline
\end{tabular}

${ }^{a}$ Solvent: THF; ${ }^{b} \mathrm{EtOH}$ as solvent; ${ }^{c}$ toluene as solvent; ${ }^{d}$ propylene carbonate as solvent; ${ }^{e}$ solvent free; ${ }^{f} \mathrm{MeOH}$ as solvent.

含有 NH 配位基，因此 Beller 课题组进一步研究发现不 包含 $\mathrm{NH}$ 基的多齿膦配体 $(\mathbf{L} 35 \sim \mathbf{L 3 7})$ 、膦氮配体 $(\mathbf{L 3 8})$ 以及氮配体(39)与金属组成的化合物在与上述几乎相同 的反应条件下 $\left(100{ }^{\circ} \mathrm{C} 、 6.0 \mathrm{MPa} \mathrm{H}_{2}\right.$ 、四氢呋喃溶剂和 $\mathrm{NaOEt}$ )没有任何反应活性(Entries $15 \sim 20$ ). 然而 L20/ 
$\mathrm{Ru}(\mathrm{acac})_{3} / \mathrm{NaOEt}$ 体系在 $\mathrm{MeOH}$ 溶剂中显示活性, 催化 草酸二甲酯生成 $96 \%$ 的乙二醇. 这些结果表明质子性溶 剂对催化反应的引发相当重要, 可能是促进活性物种的 形成, 但是文中没有深入的讨论. 催化剂 32 对草酸二甲 酯和二丁酯也都有很好的反应活性, 前者底物在 $1 \mathrm{~h}$ 、 $100{ }^{\circ} \mathrm{C}$ 下被转化得到 $84 \%$ 的乙二醇, 而后者底物在 $3 \mathrm{~h}$ 、 $60{ }^{\circ} \mathrm{C}$ 下被转化为 $95 \%$ 的乙醇酸丁酯.

2013 年, Elsiever 课题组 ${ }^{[24]}$ 合成了离子型化合物 40, 并考察了催化性能. 在 $80{ }^{\circ} \mathrm{C} 、 8.0 \mathrm{MPa} \mathrm{H}_{2}$ 、四氢呋喃溶 剂、 $\mathrm{KO}^{i} \mathrm{Bu}$ 和 $24 \mathrm{~h}$ 下催化草酸二甲酯生成 $29 \%$ 的乙醇 酸甲酯, 催化活性不佳.

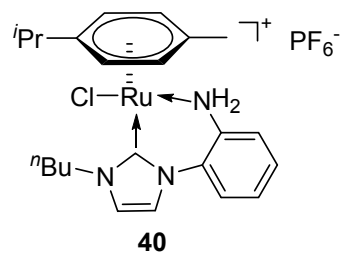

从上述的综述结果看出, 草酸酯加氢存在两种主要 的催化反应机理. Grey 和 Hanton 课题组都推测氢气在 钉中心作用下生成 $\mathrm{RuH}_{2}$, 然后 $\mathrm{RuH}_{2}$ 中的一个氢负离子 与底物分子发生加成, 由此产生的基团与另外一个氢负 离子再还原消除. 在这个过程中钓中心氧化态经历 $0 \rightarrow$ + II 的循环变化过程. 该过程被认为是金属中心主导的 “内核作用过程” (inner sphere interaction process) ${ }^{[25]}$. Beller 课题组的研究结果则确证配体 NH 以及质子性溶 剂的重要作用. Noyori ${ }^{[26]} 、$ Morris $^{[27]}$ 、Bergens ${ }^{[28]}$ 、 Saudan ${ }^{[29]}$ 、丁奎岭 ${ }^{[30]}$ 等课题组在其它羰基分子加氢反应 的研究中提出金属-NH 配体的协同作用原理, 称为 “外 部作用过程”(outer sphere proton-hydride transfer process, 经过 “六元环” 的过渡态). 与前者相比, 该过程不涉及 钉中心氧化态的变化. 同时可以看出, 在这个过程中 $\mathrm{H}_{2}$ 发生了异裂反应. 但是迄今为止尚没有人给出确丵的实 验证据证明该过程.

2014 年周其林课题组 ${ }^{[31]}$ 合成了一种空气稳定的双 吡啶四齿配体新型钓化合物 $\mathbf{4 1}$, 该化合物在温和条件 下高效催化一系列脂肪酯和芳香酯的加氢还原, 催化剂 使用量最低仅有 $0.001 \mathrm{~mol} \%$. 在 $5.1 \mathrm{MPa}$ 氢压、 $25{ }^{\circ} \mathrm{C}$ 、 异丙醇溶剂中, 反应 $16 \mathrm{~h}$ 后草酸二甲酯转化生成乙醇酸 异丙醇酯. 反应需要加入 $\mathrm{NaOMe}$. 进一步升高反应温 度至 $100{ }^{\circ} \mathrm{C}$, 反应完全转化为乙二醇. 他们推测在 $\mathrm{NaOMe}$ 和 $\mathrm{H}_{2}$ 气氛中, $\mathbf{4 1}$ 转化生成钓氢活性物种, 与底 物分子中的 $\mathrm{C}=\mathrm{O}$ 键加成, 再通过 “膦臂” $\mathrm{CH}_{2}$ 基团质 子氢转移促进单醇分子的消除; 随后醛基经过类似的氢 化过程转化为醇基. 该反应机理与 Milstein 课题组 ${ }^{[32]}$ 和 Song 课题组 ${ }^{[33]}$ 报道的结果类似, 相应的 $\mathbf{4 1}$ 被认为是一
种 “金属-配体” 双功能化作用的催化剂.

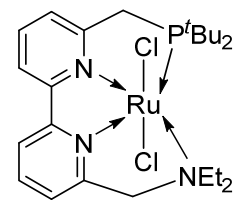

41

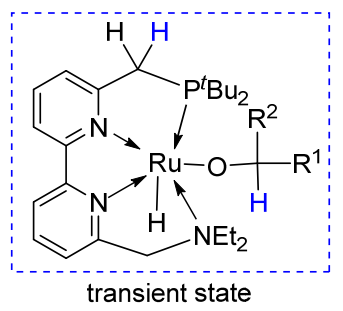

transient state
近年来我们在非均相催化草酸二甲酯为乙醇酸甲 酯和乙二醇方面开展了多方面的研究工作 ${ }^{[34]} .2016$ 年, 我们将研究从非均相拓展到均相催化反应体系, 合成了 膦氮配体钉化合物催化剂, 用于草酸二甲酯的加氢反 应. 在该研究中, 我们选择含 $\mathrm{NH}$ 基的 PN鳌合型刚性配 体与钉组成催化体系, 详细探讨了催化剂的合成和性 能(表 7) ${ }^{[35]}$.

$\mathrm{RuCl}_{2}\left(\mathrm{PPh}_{3}\right)_{3} / o-\mathrm{PPh}_{2} \mathrm{C}_{6} \mathrm{H}_{4} \mathrm{NH}_{2} / \mathrm{NaOMe}$ 体系在 100 ${ }^{\circ} \mathrm{C} 、 5.1 \mathrm{MPa} \mathrm{H}_{2}$ 、四氢呋喃溶剂中催化草酸二甲酯转化 为乙醇酸甲酯, 但是结果受金属与配体比例的影响. 当 为 $1: 1$ 时，在 $48 \mathrm{~h}$ 内产率为 $97 \% ; 1: 2$ 时 $96 \mathrm{~h}$ 内得到 相同结果; 而 $1: 3$ 时即使反应 $120 \mathrm{~h}$ 也只得到 $73 \%$ 的产 率. 这些结果暗示不同比例的反应体系可能生成不同结 构的催化活性物种.

为验证这种推测, 在催化反应温度下我们以甲苯为 溶剂分别进行了 $\mathrm{RuCl}_{2}\left(\mathrm{PPh}_{3}\right)_{3}$ 与 $o-\mathrm{PPh}_{2} \mathrm{C}_{6} \mathrm{H}_{4} \mathrm{NH}_{2}$ 按上述 计量比的反应, 在 $12 \mathrm{~h}$ 后得到单配体配位的化合物 $\mathbf{4 2}$, 而 $48 \mathrm{~h}$ 后得到双配体配位的化合物 43 . 当使用 $\mathrm{RuHCl}\left(\mathrm{PPh}_{3}\right)_{3}$ 为前驱体时, 在 $24 \mathrm{~h}$ 后得到三配体配位的 离子型化合物 44. 相应的性能考察表明, 42/NaOMe 和 $43 / \mathrm{NaOMe}$ 在上述条件下 $1 \mathrm{~h}$ 内转化生成 $97 \%$ 的乙醇酸 甲酯 $(\mathbf{4 3} / \mathrm{NaOMe}$ 也能够催化 2000 equiv. 的底物在 $16 \mathrm{~h}$ 后得到 $98 \%$ 的乙醇酸甲酯, Entry 7), 但是 $\mathbf{4 4} / \mathrm{NaOMe}$ 没 有任何活性. 这些结果显示原位组成的体系需要一定的 时间形成 PN 配体配位的钓化合物, 进而生成活性物种. 上述 $\mathrm{RuCl}_{2}\left(\mathrm{PPh}_{3}\right)_{3} / 3 o-\mathrm{PPh}_{2} \mathrm{C}_{6} \mathrm{H}_{4} \mathrm{NH}_{2} / \mathrm{NaOMe}$ 体系的催化 结果表明反应过程应该经历 $\mathbf{4 2}$ 和 $\mathbf{4 3}$ 的形成, 并逐步转 化为 44 , 最后致使反应停止, 因而底物分子不能完全转 化.

我们选择 42 与 $\mathrm{K}\left[\mathrm{HBsBu}_{3}\right]$ 反应得到了钉氢化合物 45(图 2). 同时合成了另一种双配体配位的钓化合物 46, 其与 $\mathrm{K}\left[\mathrm{HBsBu}_{3}\right]$ 反应得到另一种钓氢化合物 48. 该化合 物加热至 $70{ }^{\circ} \mathrm{C}$ 时脱去一分子氢气转化成 47 . 这样在无 $\mathrm{NaOMe}$ 的参与下 $\mathbf{4 5}$ 和 $\mathbf{4 7}$ 均能直接催化草酸二甲酯转 化为乙醇酸甲酯[产率分别是 $92 \%$ (20 h 内) 和 $86 \%$ (3 h 内)]; 添加 $\mathrm{NaOMe}$ 则提高产率至 96\%和 99\%. 
<smiles>Pc1ccccc1N[Te](Cl)(Cl)(Pc1ccccc1)c1ccccc1</smiles>

42

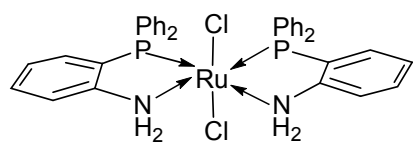

43
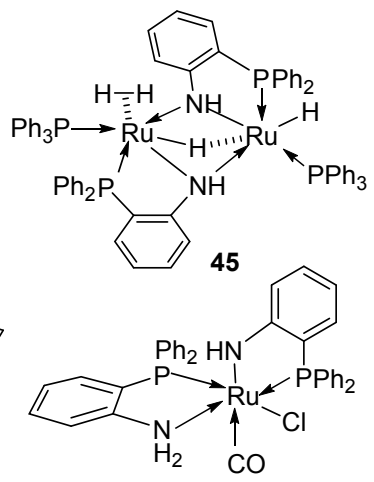

46
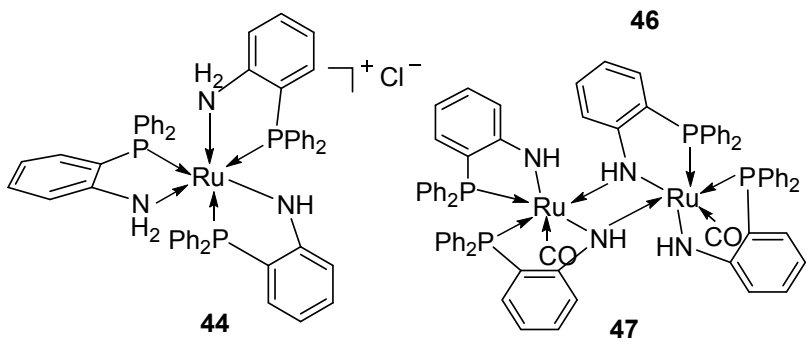

表 $7 \mathrm{PN}$ 配体钉催化体系催化 $\mathrm{DMO}$ 加氢转化为 $\mathrm{MG}$ 的反应 结果 ${ }^{a}$

Table 7 Results for hydrogenation of DMO to MG catalyzed by PN-ligand coordinated ruthenium catalysts

\begin{tabular}{|c|c|c|c|c|c|}
\hline \multirow{2}{*}{ Entry } & \multirow{2}{*}{ Cat. } & \multirow{2}{*}{$T /{ }^{\circ} \mathrm{C}$} & \multirow{2}{*}{$t / \mathrm{h}$} & \multicolumn{2}{|c|}{ Yield/\% } \\
\hline & & & & MG & $\mathrm{EQ}$ \\
\hline 1 & $\mathrm{RuCl}_{2}\left(\mathrm{PPh}_{3}\right)_{3} / \mathrm{NaOMe}$ & 100 & 1 & 0 & 0 \\
\hline 2 & $\begin{array}{l}\mathrm{RuCl}_{2}\left(\mathrm{PPh}_{3}\right)_{3} / o-\mathrm{PPh}_{2^{-}} \\
\mathrm{C}_{6} \mathrm{H}_{4} \mathrm{NH}_{2} / \mathrm{NaOMe}\end{array}$ & 100 & 48 & 97 & 0 \\
\hline 3 & $\begin{array}{l}\mathrm{RuCl}_{2}\left(\mathrm{PPh}_{3}\right)_{3} / 2 o-\mathrm{PPh}_{2}- \\
\mathrm{C}_{6} \mathrm{H}_{4} \mathrm{NH}_{2} / \mathrm{NaOMe}\end{array}$ & 100 & 96 & 97 & 0 \\
\hline 4 & $\begin{array}{l}\mathrm{RuCl}_{2}\left(\mathrm{PPh}_{3}\right)_{3} / 3 o-\mathrm{PPh}_{2}- \\
\mathrm{C}_{6} \mathrm{H}_{4} \mathrm{NH}_{2} / \mathrm{NaOMe}\end{array}$ & 100 & 120 & 73 & 0 \\
\hline 5 & 42 $/ \mathrm{NaOMe}$ & 100 & 1 & 97 & 0 \\
\hline 6 & 43/NaOMe & 100 & 1 & 97 & 0 \\
\hline 7 & $43 / \mathrm{NaOMe}^{c}$ & 25 & 16 & 98 & 0 \\
\hline 8 & 44/NaOMe & 100 & 1 & 0 & 0 \\
\hline 9 & 45 & 100 & 20 & 92 & 0 \\
\hline 10 & 45/NaOMe & 100 & 20 & 96 & 0 \\
\hline 11 & 47 & 100 & 3 & 86 & 0 \\
\hline 12 & 47/ $\mathrm{NaOMe}$ & 100 & 3 & 99 & 0 \\
\hline
\end{tabular}

${ }^{a}$ Solvent: THF, NaOMe: 10 equiv, DMO: 200 equiv, $p\left(\mathrm{H}_{2}\right): 5.1 \mathrm{MPa} .{ }^{c} 2000$ equiv. DMO.

化合物 47 和 48 可以相互转化(Eq. 1, 图 3), 不难发 现 $\mathrm{H}_{2}$ 分子的脱除源自 $\mathrm{RuH}$ 和配体中的 $\mathrm{NH}_{2}$ 基团的作用, 即两基团从键接状态 $\mathrm{HRu} \leftarrow \mathrm{NH}_{2}$ 变为 $\mathrm{RuNH}$ (后者易于 二聚), 而 $\mathrm{RuNH}$ 与 $\mathrm{H}_{2}$ 反应又转回 $\mathrm{HRu} \leftarrow \mathrm{NH}_{2}$ 键接状态 (注: Noyori 等课题组将这种转换定义为 $\mathrm{Ru}-\mathrm{N}$ 单键和 双键之间的变化. 我们采用单键和配位键的变化意在表 明 $\mathrm{Ru}$ 中心的氧化态保持不变, 与 “内核作用原理” 不 同). $\mathrm{D}_{2}$ 实验显示这种转化即使在 $10{ }^{\circ} \mathrm{C}$ 时仍然进行.

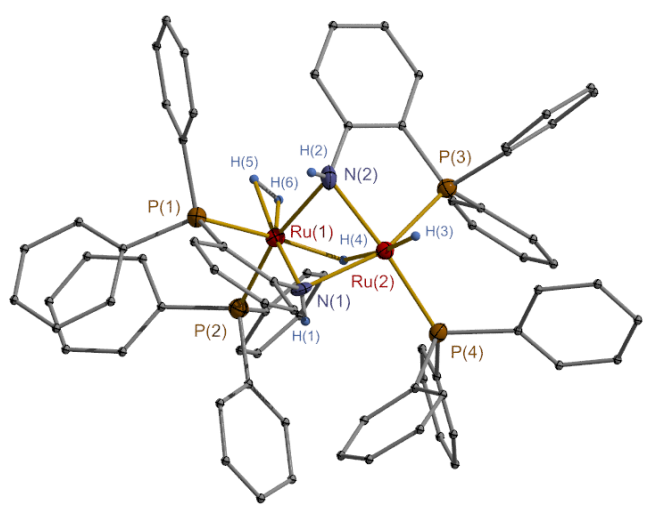

图 2 化合物 $\mathbf{4 5}$ 的单晶结构

Figure $2 \mathrm{X}$ ray crystal structure of catalyst $\mathbf{4 5}$
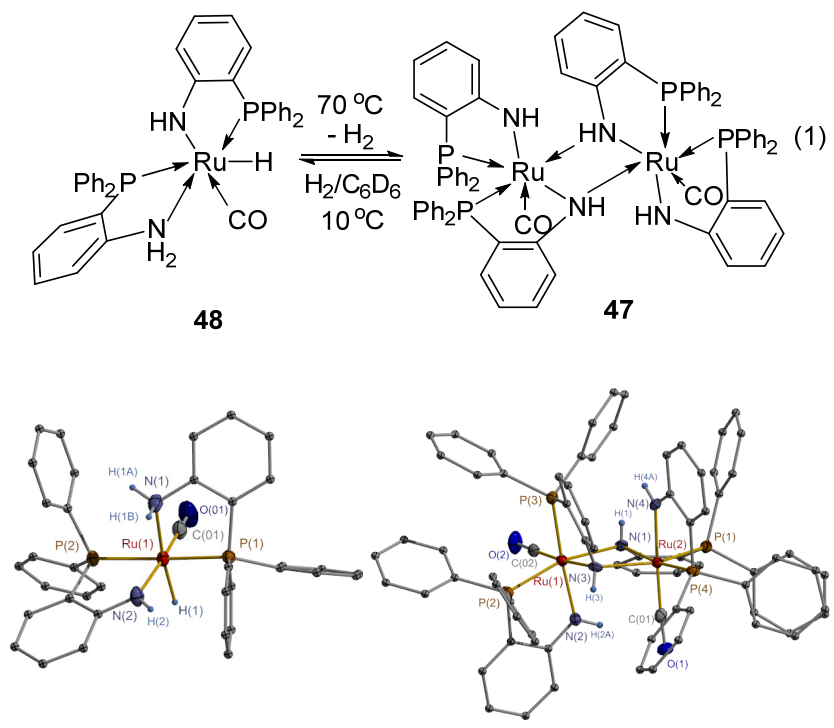

图 3 化合物 48 (左) 和 47 (右) 的单晶结构

Figure 3 X-ray crystal structures of complexes 48 (left) and 47 (right)

这样，我们对上述催化反应机理进行了推测. 如 Scheme 6 所示, 处于 $\mathrm{HRu} \leftarrow \mathrm{NH}_{2}$ 键接状态的催化活性物 种 48 与底物分子中的 $\mathrm{C}=\mathrm{O}$ 通过六元环过渡态的电性 作用引发氢负离子-质子转移, 产生半缩酫分子, 其重 排脱去一分子的甲醇生成乙醛酸甲酯; 该分子中醛基 $\mathrm{C}=\mathrm{O}$ 再与 48 经上述作用生成乙醇酸甲酯产物. 氢负离 子-质子迁移后的钓物种易于二聚形成 47,47 在反应过 程中可看成是一种 “稳态”, 其进一步与 $\mathrm{H}_{2}$ 反应重新形 成活性态 48.

进一步研究发现调变反应条件时, $42 / \mathrm{NaOMe}$ 和 43/ $/ \mathrm{NaOMe}$ 都能催化草酸二甲酯到乙二醇. 以上述反应 条件为基础，提高一倍 $\mathrm{NaOMe}$ 量、升高温度至 $120{ }^{\circ} \mathrm{C}$ 、 $\mathrm{DMO}$ 量减半时, 42 在 $36 \mathrm{~h}$ 后催化生成 $31 \%$ 的乙醇酸甲 酯和 $61 \%$ 的乙二醇; 在 $72 \mathrm{~h}$ 后生成 $25 \%$ 的乙醇酸甲酯和 $68 \%$ 的乙二醇; 进一步升高温度至 $140{ }^{\circ} \mathrm{C}$ 在 $36 \mathrm{~h}$ 后使乙 醇酸甲酯完全转化得到 $94 \%$ 的乙二醇. 使用催化剂 $\mathbf{4 3}$, 


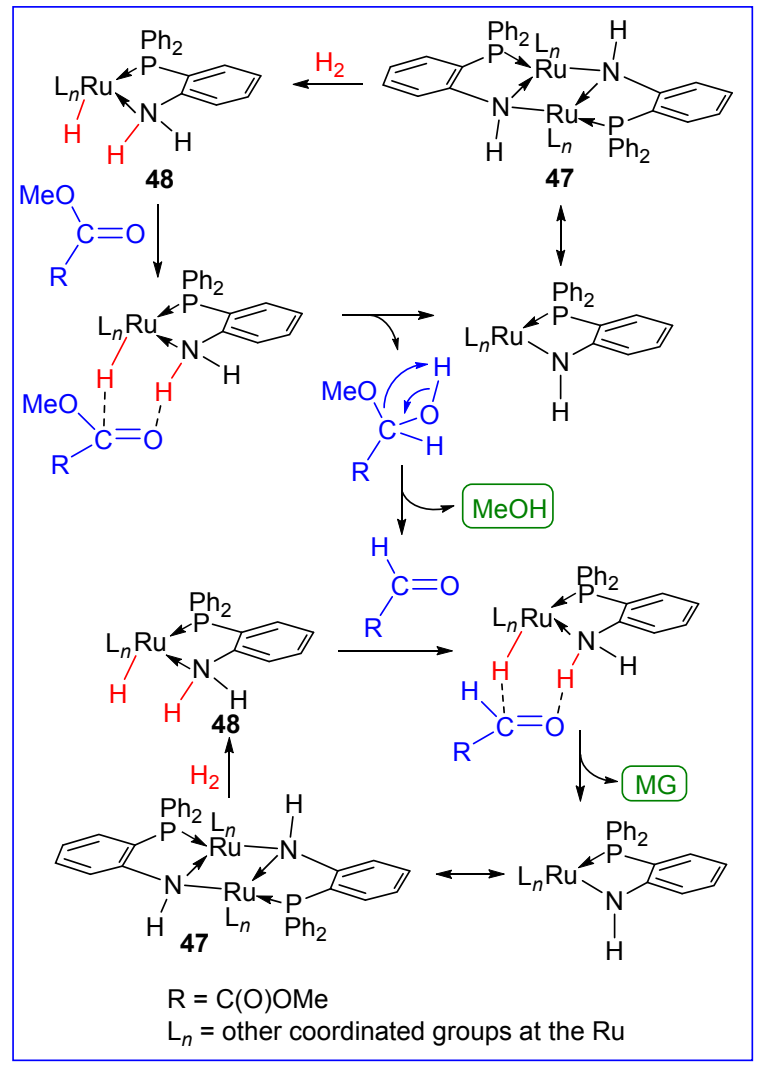

图式 6 推测的反应机理

Scheme 6 Suggested mechanism

在 $120{ }^{\circ} \mathrm{C} 、 36 \mathrm{~h}$ 后乙醇酸甲酯转化完全得到 $97 \%$ 的乙 二醇.

表 $8 \mathrm{PN}$ 配体钉催化体系催化 $\mathrm{DMO}$ 加氢转化为 $\mathrm{MG}$ 和 $\mathrm{EG}$ 的反应结果 ${ }^{a}$

Table 8 Results for hydrogenation of DMO to MG and EG catalyzed by PN-ligand coordinated ruthenium catalysts

\begin{tabular}{cccccc}
\hline \multirow{2}{*}{ Entry } & \multirow{2}{*}{ Cat. } & \multirow{2}{*}{$T /{ }^{\circ} \mathrm{C}$} & \multirow{2}{*}{$t / \mathrm{h}$} & \multicolumn{2}{c}{ Yield/\% } \\
\cline { 5 - 7 } & & & & MG & EG \\
\hline 1 & $\mathbf{4 2} / 20 \mathrm{NaOMe}$ & 120 & 36 & 31 & 61 \\
2 & $\mathbf{4 2} / 20 \mathrm{NaOMe}$ & 120 & 72 & 25 & 68 \\
3 & $\mathbf{4 2} / 20 \mathrm{NaOMe}$ & 140 & 36 & 0 & 94 \\
4 & $\mathbf{4 3} / 20 \mathrm{NaOMe}$ & 120 & 16 & 51 & 47 \\
5 & $\mathbf{4 3} / 20 \mathrm{NaOMe}$ & 120 & 24 & 20 & 75 \\
6 & $\mathbf{4 3} / 20 \mathrm{NaOMe}$ & 100 & 36 & 0 & 97 \\
\hline
\end{tabular}

${ }^{a}$ Solvent: THF, DMO: 100 equiv., $p\left(\mathrm{H}_{2}\right): 5.1 \mathrm{MPa}$.

显而易见，使用同一种钓化合物 $\mathbf{4 2}$ 或 43 作催化剂 在催化得到乙二醇时需要更为苛刻的条件. 这也印证了 草酸二甲酯的反应活性要强于乙醇酸甲酯. 升高反应温 度是促进直接转化成乙二醇的一种有效途径, 但是使用 更多量的 $\mathrm{NaOMe}$ 亦可显著提高乙二醇的生成. 值得注 意的是，该反应是在封闭体系中进行，甲醇是体系中的 副产物. 从动力学角度考虑, 生成乙二醇会产生两个分 子的甲醇. 这样生成乙二醇的反应要比生成乙醇酸甲酯 的难很多. 对比也会发现, 使用同一种催化剂, 转化为
乙二醇产物的时间要比转化为乙醇酸甲酯的时间长得 多. 乙二醇的生成机理类似于 Scheme 6 , 只是将其中草 酸二甲酯底物换为乙醇酸甲酯. 在含 $\mathrm{NH}$ 基团的 PN 配体 钓化合物催化剂体系中, 我们确定了重要化合物 48 和 47 的结构, 揭示了这两个化合物之间内含的 $\mathrm{H}_{2}$ 分子转 换, 证实了它们的催化性能, 这些为 “金属-NH 配体” 协同的 “外部催化作用机理” 提供了可靠的实验证据.

\section{3 总结与展望}

草酸酯的钉金属均相催化加氢反应是一个明确的 溶液均相态的分子反应过程, 其中关键的步骤涉及到 $\mathrm{H}_{2}$ 分子的活化进而加成到底物分子中的 $\mathrm{C}=\mathrm{O}$ 上. 使用 配体钓化合物为催化剂, 配体必需具有强的配位能力来 稳定钉中心发生官能化作用. 对于 “外部氢负离子-氢质 子转移作用过程”，配体包含协同金属中心异裂 $\mathrm{H}_{2}$ 的基 团为佳, 否则要使用质子性的溶剂来协同金属中心作 用. 反应条件对反应的进行和转化相当重要. 温度决定 分子碰撞程度, 升高温度有利于反应速率的提高, 但是 过高的温度也会引起产物的分解以及其它副反应. 氢压 决定 $\mathrm{H}_{2}$ 分子在溶液中的浓度, 高 $\mathrm{H}_{2}$ 压有利于反应向产 物方向的动力学转化. 碱性添加剂如醇钠会促进钉中心 特别是含氯基团生成活性氢负离子基，同时它也会对配 体中含 $\mathrm{N}$ 基团的亲核性有重要影响 ${ }^{[28]}$. 使用不同酯底物 时，醇钠有选择性，如对于草酸二甲酯，通常使用甲醇 钠，而对于草酸二乙酯则使用乙醇钠等. 金属锌添加剂 在针对乙酰丙酮钓前驱体时, 具有很好地促进三价钓还 原的作用.

在这个反应体系中, 乙醇酸甲酯和乙二醇都是重要 的目标产物. 对决大多数反应来说, 同一种催化剂都具 有转化生成这两种产物的催化能力, 但是由于草酸酯和 乙醇酸甲酯的反应性差异, 乙醇酸甲酯生成比较容易, 进一步转化成乙二醇需要调变反应条件, 这个调变需要 在保证产物不分解的前提下才有意义. 从反应工艺上 讲，目前的催化反应多在反应釜密闭体系中进行，体系 中会产生单醇副产物, 从反应体系中移出产物和副产物 将会大大提高反应效率. 如能在反应工艺中实现这个操 作, 均相催化反应体系将不失为一个极具潜力的应用体 系, 因为该体系反应条件要较非均相体系温和很多, 而 且反应效率高，并能有效地控制乙醇酸甲酯和乙二醇的 高选择性产出.

\section{References}

[1] (a) Jiang, Z. Synthetic Technology and Application 2010, 25(4), 27 (in Chinese).

(江镇海, 合成技术及应用, 2010, 25(4), 27.)

(b) Huang, G.; Li, X.; Yang, Y.; Qu, J. Petrochem. Technol. Appl. 2015, 33, 75 (in Chinese). 
(黄格省, 李雪静, 杨延翔, 曲静波, 石化技术与应用, 2015, 33, 75.)

[2] (a) Li, D.; Wang, H. Modern Chem. Ind. 2017, 37(1), 5 (in Chinese).

(李代红，王洪波，现代化工，2017, 37(1), 5.)

(b) Liu, Z. Chem. Ind. Eng. Proc. 2013, 32, 1214 (in Chinese).

(刘宗语, 化工进展, 2013, 32, 1214.)

(c) Chen, W.; Sun, J.; Zhang, J.; Zhang, S.; Hua, W. Chem. Ind. Eng. Proc. 2014, 33, 1740 (in Chinese).

(成卫国, 孙剑, 张军平, 张锁江, 华炜, 化工进展, 2014, 33, 1740.)

[3] (a) Gaylord, N. G. J. Chem. Educ. 1957, 34, 367.

(b) Hudlicky, M. Reductions in Organic Chemistry, ACS, Washington, DC, 1996.

[4] (a) Modler, R. F.; Gubler, R.; Inoguchi, Y. Detergent Alchols, CEH Marketing Research Report, 2004.

(b) Hong, W. S. Natural Detergent Alcohols by a Vapour Phase Ester Hydrogenation Process, PPP Review 93-2-1, 2004.

(c) Cant, N. W.; Trimm, D. L.; Turek, T. Catal. Rev. Sci. Eng. 1994, 36,645 .

[5] March, J. Advanced Organic Chemistry: Reactions Mechanisms and Structure, 4th ed., Wiley-Interscience, New York, 1992, p. 1213.

[6] (a) Rylander, P. N. Catalytic Hydrogenation in Organic Syntheses, Academic Press, New York, 1979.

(b) de Vries, J. G.; Elsevier, C. J. Handbook of Heterogeneous Hydrogenation for Organic Synthesis, Weily-VCH, Weinheim, 2007.

[7] (a) Yue, H.-R.; Zhao, Y.-J.; Ma, X.-B.; Gong, J.-L. Chem. Soc. Rev. 2012, 41,4218 .

(b) Zhao, Y.; Zhao, S.; Wang, B.; Lv, J.; Ma, X. Chem. Indu. Eng. Prog. 2013, 32, 721 (in Chinese).

(赵玉军, 赵硕, 王博, 吕静, 马新宾, 化工进展, 2013, 32, 721.)

(c) Jin, E.-L.; Zhang, Y.-L.; He, L.-L.; Harris, H. G.; Teng, B.-T.; Fan, M.-H. Appl. Catal. A: Gen. 2014, 476, 158

(d) Higman, C.; Tam, S. Chem. Rev. 2014, 114, 1673.

(e) Li, J.; Duan, X.; Lin, H.; Ye, L.; Yuan, Y. Petrochem. Technol. 2014, 43, 985 (in Chinese).

(李建辉，段新平，林海强，叶林敏，袁友珠，石油化工，2014, 43, 985.)

(f) Li, S.-M.; Wang, Y.; Zhang, J.; Wang, S.-P.; Xu, Y.; Zhao, Y.-J.; Ma, X.-B. Ind. Eng. Chem. Res. 2015, 54, 1243.

[8] (a) Bayón, J. C.; Claver, C.; Masdeu-Bultó, A. M. WO 2003093208, 2003 [Chem. Abstr. 2003, 139, 366612].

(b) Pope, S. J. A.; Champness, N. R.; Reid, G. J. Chem. Soc., Dalton Trans. 1997, 1639.

[9] Seyden-Penne, J. Reductions by the Allumino- and Borohydride in Organic Synthesis, 2nd ed.; Wiley-VCH, New York, 1997.

[10] (a) Turek, T.; Trimm, D.; Cant, N. Catal. Rev. -Sci. Eng. 1994, 36, 645.

(b) Pouilloux, Y.; Autin, F.; Barrault, J. Catal. Today 2000, 63, 87.

[11] Pritchard, J.; Filonenko, G. A.; van Putten, R.; Hensen, E. J. M.; Pidko, E. A. Chem. Soc. Rev. 2015, 44, 3808.

[12] (a) Grey, R. A.; Pez, G. P.; Wallo, A.; Corsi, J. J. Chem. Soc., Chem. Commun. 1980, 783.

(b) Grey, R. A.; Pez, G. P.; Wallo, A. J. Am. Chem. Soc. 1981, 103, 7536.

(c) De Graauw, C. F.; Peters, J. A.; van Bekkum, H.; Huskens, J. Synthesis 1994, 1007.

(d) Ashby, E. C. Acc. Chem. Res. 1988, 21, 414.

[13] Matteoli, U.; Bianchi, M.; Menchi, G.; Frediani, P.; Piacenti, F. J. Mol. Catal. 1984, 22, 353.

[14] (a) Matteoli, U.; Bianchi, M.; Menchi, G.; Frediani, P.; Piacenti, F. J. Mol. Catal. 1985, 29, 269

(b) Matteoli, U.; Menchi, G.; Bianchi, M.; Piacenti, F. J. Organomet. Chem. 1986, 299, 233.

(c) Matteoli, U.; Bianchi, M.; Menchi, G.; Piacenti, F. J. Mol. Catal. 1988, 44,347

[15] (a) Matteoli, U.; Bianchi, M.; Menchi, G.; Piacenti, F. J. Mol. Catal.
1991, 64, 257.

(b) Matteoli, U.; Menchi, G.; Bianchi, M.; Piacenti, F.; Ianelli, S.; Nardelli, M. J. Organomet. Chem. 1995, 498, 177.

[16] Teunissen, H. T.; Elsevier, C. J. Chem. Commun. 1997, 667.

[17] Kara, Y.; Wada, K. Chem. Lett. 1991, 20, 553.

[18] van Engelen, M. C.; Teunissen, H. T.; de Vries, J. G.; Elsevier, C. J. J. Mol. Catal. A: Chem. 2003, 206, 185

[19] Boardman, B.; Hanton, M. J.; van Rensburg, H.; Tooze, R. P. Chem. Commun. 2006, 2289.

[20] Bayón, J. C.; Claver, C.; Masdeu-Bultó, A. M. Coord. Chem. Rev. 1999, $193 \sim 195,73$

[21] Hanton, M. J.; Tin, S.; Boardman, B. J.; Miller, P. J. Mol. Catal. A: Chem. 2011, 346, 70.

[22] Ziebart, C.; Jackstell, R.; Beller, M. ChemCatChem 2013, 5, 3228.

[23] Kuriyama, W.; Matsumoto, T.; Ogata, O.; Ino, Y.; Aoki, K.; Tanaka, S.; Ishida, K.; Kobayashi, T.; Sayo, N.; Saito, T. Org. Process Res. Dev. 2012, 16, 166.

[24] Eveline, J.; Jongbloed, L. S.; Tromp, D. S.; Martin, L.; Bas, D. B.; Elsevier, C. J. ChemSusChem 2013, 6, 1737.

[25] (a) Bryndza, H. E.; Tam, W. Chem. Rev. 1988, 88, 1163.

(b) Pàmies, O.; Bäckvall, J. E. Chem. Eur. J. 2001, 7, 5052.

(c) Fulton, J. R.; Holland, A. W.; Fox, D. J.; Vergman, R. G. Acc. Chem. Res. 2002, 35, 44.

(d) Clapham, S. E.; Hadzovic, A.; Morris, R. H. Coord. Chem. Rev. 2004, 248, 2201.

[26] (a) Yamakawa, M.; Ito, H.; Noyori, R. J. Am. Chem. Soc. 2000, 122, 1466 .

(b) Noyori, R.; Ohkuma, T. Angew. Chem. Int. Ed. 2001, 40, 40.

(c) Ohkuma, T.; Koizumi, M.; Muñiz, K.; Hilt, G.; Kabuto, C.; Noyori, R. J. Am. Chem. Soc. 2002, 124, 6508.

(d) Sandoval, C. A.; Ohkuma, T.; Muñiz, K.; Noyori, R. J. Am. Chem. Soc. 2003, 125, 13490.

[27] (a) Abdur-Rashid, K.; Clapham, S. E.; Hadzovic, A.; Harvey, J. N.; Lough, A. J.; Morris, R. H. J. Am. Chem. Soc. 2002, 124, 15104.

(b) Abdur-Rashid, K.; Faatz, M.; Lough, A. J.; Morris, R. H. J. Am. Chem. Soc. 2001, 123, 7473.

(c) Abbel, R.; Abdur-Rashid, K.; Faatz, M.; Hadzovic, A.; Lough, A. J.; Morris, R. H. J. Am. Chem. Soc. 2005, 127, 1870.

(d) Abdur-Rashid, K.; Guo, R.; Lough, A. J.; Morris, R. H.; Song, D. Adv. Synth. Catal. 2005, 347, 571 .

[28] (a) Hamilton, R. J.; Bergens, S. H. J. Am. Chem. Soc. 2006, 128, 13700 .

(b) Hamilton, R. J.; Bergens, S. H. J. Am. Chem. Soc. 2008, 130, 11979 .

(c) Takebayashi, S.; Dabral, N.; Miskolzie, M.; Bergens, S. H. J. Am. Chem. Soc. 2011, 133, 9666.

(d) John, J. M.; Takebayashi, S.; Dabral, N.; Miskolzie, M.; Bergens, S. H. J. Am. Chem. Soc. 2013, 135, 8578.

[29] Saudan, L. A.; Saudan, C. M.; Debieux, C.; Wyss, P. Angew. Chem., Int. Ed. 2007, 46, 7473.

[30] (a) Han, Z.; Rong, L.; Wu, J.; Zhang, L.; Wang, Z.; Ding, K. Angew. Chem., Int. Ed. 2012, 51, 13041.

(b) Zhao, B.; Han, Z.; Ding, K. Angew. Chem., Int. Ed. 2013, 52, 4744.

[31] Li, W.; Xie, J.; Yuan, M.; Zhou, Q. Green Chem. 2014, 16, 4081.

[32] Zhang, J.; Leitus, G.; Ben-David, Y.; Milstein, D. Angew. Chem., Int. Ed. 2006, 45, 1113.

[33] (a) Sun, Y.; Koehler, C.; Tan, R.; Annibale, V. T.; Song, D. Chem. Commun. 2011, 47, 8349.

(b) Yang, X. ACS Catal. 2012, 2, 964

[34] (a) He, Z.; Lin, H.; He, P.; Yuan, Y. J. Catal. 2011, 277, 54.

(b) Wang, Y.; Duan, X.; Zheng, J.; Lin, H.; Yuan, Y.; Ariga, H.; Takakusagi, S.; Asakura, K. Catal. Sci. Technol. 2012, 2, 1637.

(c) Huang, Y.; Ariga, H.; Zheng, X.; Duan, X.; Takakusagi, S.; Asakura, K.; Yuan, Y. J. Catal. 2013, 307, 74.

(d) Zheng, J.; Lin, H.; Wang, Y.; Zheng, X.; Duan, X.; Yuan, Y. J. Catal. 2013, 297, 110 .

(e) Zheng, X.; Lin, H.; Zheng, J.; Duan, X.; Yuan, Y. ACS Catal. 
2013, 3, 2738.

(f) Li, M.; Ye, L.; Zheng, J.; Fang, H.; Kroner, A.; Yuan, Y.; Tsang, S. C. E. Chem. Commun. 2016, 52, 2569.

(g) Li, M.; Zheng, J.; Qu, J.; Liao, F.; Raine, E.; Kuo, W. C. H.; Su, S. S.; Po, P.; Yuan, Y.; Tsang, S. C. E. Sci. Rep. 2016, 6, 20527. (h) Zheng, J.; Duan, X.; Lin, H.; Gu, Z.; Fang, H.; Li, J.; Yuan, Y. Nanoscale 2016, 8, 5959.

[35] Fang, X.; Zhang, C.; Chen, J.; Zhu, H.; Yuan, Y. RSC Adv. 2016, 6, 45512 .

(Li, L. T.) 